\title{
Nuclear organisation of some immunohistochemically identifiable neural \\ systems in three Afrotherian species-Potomogale velox, Amblysomus \\ hottentotus and Petrodromus tetradactylus
}

\section{Tanya Calvey ${ }^{1}$, Nina Patzke ${ }^{1}$, Consolate Kaswera ${ }^{2}$, Emmanuel Gilissen ${ }^{3,4,5}$, Nigel C. Bennett $^{6} \&$ Paul R. Manger ${ }^{1}$}

\footnotetext{
${ }^{1}$ School of Anatomical Sciences, Faculty of Health Sciences, University of the Witwatersrand, 7 York Road, Parktown 2193, Johannesburg, Republic of South Africa.

${ }^{2}$ Faculté des Sciences, University of Kisangani, B.P 1232 Kisangani, Democratic Republic of Congo.

${ }^{3}$ Department of African Zoology, Royal Museum for Central Africa, Leuvensesteenweg 13, B3080 Tervuren, Belgium.

${ }^{4}$ Laboratory of Histology and Neuropathology, Université Libre de Bruxelles, 1070 Brussels, Belgium.

${ }^{5}$ Department of Anthropology, University of Arkansas, Fayetteville, AR 72701, USA.

${ }^{6}$ Mammal Research Institute, Department of Zoology and Entomology, University of Pretoria, Pretoria 0002, South Africa.
}

Correspondence to: Paul Manger

School of Anatomical Sciences

Faculty of Health Sciences

University of the Witwatersrand

7 York Road, Parktown, 2193

Johannesburg, REPUBLIC OF SOUTH AFRICA.

Ph: +27 117172497 Fax: +27 117172422

Paul.Manger@wits.ac.za 


\title{
Highlights
}

- The organisation of four neural systems is described in the brains of three Afrotherians not previously studied

- These systems are organised in a similar manner to other mammals.

- There are features that reflect both phylogenetic and functional signals in the evolution of these neural systems.

- The golden mole appears to be a "hyper-cholinergic" mammal, possessing cholinergic neurons in novel regions of the brain.

\begin{abstract}
The present study describes the organization of the cholinergic, catecholaminergic, serotonergic and orexinergic (hypocretinergic) neurons in the brains of the giant otter shrew, the Hottentot golden mole and the four-toed sengi, three members of the mammalian super order Afrotheria. The aim of the present study was to investigate the possible differences in the nuclear complement of these neural systems in comparison to previous studies on other Afrotheria species and other mammalian species. Brains of the golden mole, sengi and giant otter shrew were coronally sectioned and immunohistochemically stained with antibodies against cholineacetyl-transferase, tyrosine hydroxylase, serotonin and orexin-A. The majority of nuclei revealed in the current study were similar between the species investigated, to other Afrotherian species investigated, and to other mammals, but certain differences in the nuclear complement highlighted phylogenetic interrelationships. The golden mole was seen to have cholinergic interneurons in the cerebral cortex, hippocampus, olfactory bulb and amygdala. The four-toed sengi had cholinergic neurons in both colliculi and in the cochlear nucleus, but lacked the catecholaminergic A15d group in the hypothalamus. In both the golden mole and the four-toed sengi, the locus coeruleus (A6d group) was made up of few neurons. The golden mole also exhibited an unusual foreshortening of the brain, such that a major kink in the brainstem was evident. The results of this study, framed in a phylogenetic context, appear to indicate that the golden mole and four-toed sengi share a more recent common ancestor that diverged from the tenrec lineage early in the phylogenetic history of the Afrotherians.
\end{abstract}

Keywords: Choline acetyltransferase; Tyrosine hydroxylase; Serotonin; Hypocretin; Orexin; Evolution; Afrotherian 


\footnotetext{
Abbreviations

III - oculomotor nucleus

IV - trochlear nucleus

Vmot - motor division of trigeminal nerve nucleus

Vsens - sensory division of trigeminal nerve nucleus

VI - abducens nucleus

VIId - dorsal division of facial nerve nucleus

VIIv - ventral division of facial nerve nucleus

$\mathrm{X}$ - dorsal motor vagus nucleus

XII - hypoglossal nucleus

$3 \mathrm{~V}-$ third ventricle

$4 \mathrm{~V}$ - fourth ventricle

5n - trigeminal nerve

$7 \mathrm{n}$ - facial nerve

A1 - caudal ventrolateral medullary tegmental nucleus

A2 - caudal dorsomedial medullary nucleus

A4 - dorsolateral division of locus coeruleus

A5 - fifth arcuate nucleus

A6c-compact portion of locus coeruleus

A6d - diffuse portion of locus coeruleus

A7d - nucleus subcoeruleus, diffuse portion

A7sc - nucleus subcoeruleus, compact portion

A8 - retrorubral nucleus

A91 - substantia nigra, lateral

A9m - substantia nigra, medial

A9pc - substantia nigra, pars compacta

A9v - substantia nigra, ventral, pars reticulata

A10 - ventral tegmental area
} 
A10c - ventral tegmental area, central

A10d - ventral tegmental area, dorsal

A10dc - ventral tegmental area, dorsal caudal

A11 - caudal diencephalic group

A12 - tuberal cell group

A13 - zona incerta cell group

A14 - rostral periventricular nucleus

A15d - anterior hypothalamic group, dorsal division

A15v - anterior hypothalamic group, ventral division

A16 - catecholaminergic neurons of the olfactory bulb

$\mathrm{ac}$ - anterior commissure

$\mathrm{ACN}$ - cholinergic neurons of the amygdala

Amyg - amygdala

$\mathrm{AON}$ - anterior olfactory nucleus

$\mathrm{AP}$ - area postrema

B9 - supralemniscal serotonergic nucleus

bic - brachium of the inferior colliculus

$\mathrm{C} 1$ - rostral ventrolateral medullary tegmental group

C2 - rostral dorsomedial medullary nucleus

C3 - rostral dorsal midline medullary nucleus

$\mathrm{C}$ - caudate nucleus

ca - cerebral aqueduct

$\mathrm{Cb}$ - cerebellum

cc - corpus callosum

$\mathrm{CCN}$ - cholinergic neurons of the cerebral cortex

$\mathrm{Cl}$ - claustrum

$\mathrm{CLi}$ - caudal linear nucleus

$\mathrm{CN}$ - deep cerebellar nucleus 
Co - cochlear nuclei

$\mathrm{CoCN}$ - cholinergic neurons of the cochlear nuclear complex

csc - commissure of the superior colliculi

CVL - caudal ventrolateral serotonergic group

dh - dorsal horn of spinal cord

df - dorsal funiculus of spinal cord

Diag. B - diagonal band of Broca

$\mathrm{DRc}$ - dorsal raphe, caudal division

DRd - dorsal raphe, dorsal division

DRif - dorsal raphe, interfascicular division

DRl - dorsal raphe, lateral division

DRp - dorsal raphe, peripheral division

$\mathrm{DRv}$ - dorsal raphe, ventral division

DT - dorsal thalamus

EPL - external plexiform layer of olfactory bulb

EW - Edinger-Westphal nucleus

f- fornix

$\mathrm{fr}-$ fasciculus retroflexus

GC - central grey matter

GCLi - inner granular cell layer of olfactory bulb

GCLo - outer granular cell layer of olfactory bulb

GL - glomerular layer of olfactory bulb

GP - globus pallidus

$\mathrm{Hbc}$ - habenular commissure

$\mathrm{Hbl}$ - lateral habenular nucleus

Hbm - medial habenular nucleus

hc - hippocampal commissure

$\mathrm{HCN}$ - cholinergic neurons of the hippocampus

HIP - hippocampus 
Hyp - hypothalamus

Hyp.d - dorsal hypothalamic cholinergic nucleus

Hyp.1 - lateral hypothalamic cholinergic nucleus

Hyp.v - ventral hypothalamic cholinergic nucleus

IC - inferior colliculus

ic - internal capsule

ICCN - cholinergic neurons of the inferior colliculus

io - inferior olivary nucleus

IP - interpeduncular nucleus

Is.Call/TOL - islands of Calleja and the olfactory tubercle

LDT - laterodorsal tegmental nucleus

LGv - ventral lateral geniculate nucleus

LOT - lateral olfactory tract

LV - lateral ventricle

$\mathrm{MB}$ - mammillary body

$\mathrm{Mc}$ - main cluster of orexinergic neurons

MCL - mitral cell layer of olfactory bulb

mcp - middle cerebellar peduncle

$\mathrm{MnR}$ - median raphe nucleus

mlf - medial longitudinal fasciculus

mtf - medullary tegmental field

N.Acc - nucleus accumbens

N.Amb - nucleus ambiguus

N.Bas - nucleus basalis

NEO - cerebral neocortex

OC - optic chiasm

OCN - cholinergic neurons of olfactory bulb

$\mathrm{ONL}$ - olfactory nerve layer of olfactory bulb

OT - optic tract 
OTc - optic tract cluster of orexinergic neurons

$\mathrm{OV}$ - olfactory ventricle

$\mathrm{P}$ - putamen nucleus

$\mathrm{PBg}$ - parabigeminal nucleus

$\mathrm{PC}$ - cerebral peduncle

$\mathrm{pc}$ - posterior commissure

PCCN - cholinergic neurons of the piriform cortex

PIR - piriform cortex

PPT - pedunculopontine nucleus

PTa - pretectal area

py - pyramidal tract

$\mathrm{PVG}$ - periventricular grey matter

PVL - periventricular layer of olfactory bulb

pVII - preganglionic motor neurons of the superior salivatory nucleus or facial nerve

pIX - preganglionic motor neurons of the inferior salivatory nucleus

$\mathrm{R}$ - reticular nucleus of the dorsal thalamus

Rmc - magnocellular division of red nucleus

$\mathrm{RMg}$ - raphe magnus nucleus

$\mathrm{ROb}$ - raphe obscurus nucleus

$\mathrm{RPa}$ - raphe pallidus nucleus

RVL - rostral ventrolateral serotonergic group

$\mathrm{S}$ - septal nuclear complex

$\mathrm{SC}$ - superior colliculus

SCCN - cholinergic neurons of the superior colliculus

scp - superior cerebellar peduncle

Sep.L - lateral septal nucleus

Sep.M - medial septal nucleus

so- superior olivary nucleus

$\mathrm{spV}$ - spinal trigeminal tract 
vh - ventral horn of spinal cord

VPO - ventral pontine nucleus

xscp - decussation of the superior cerebellar peduncle

$\mathrm{zi}$ - zona incerta

Zic - zona incerta cluster of orexinergic neurons

\section{Introduction}

The Afrotherian mammalian cohort is comprised of what may be considered to be a very unusual mammalian grouping, one whose relationships have mainly been resolved on molecular rather than morphological grounds (e.g. van Dijk et al., 2001; Arnason et al., 2008; Hallström and Janke, 2008; Prasad et al., 2008; Asher et al., 2010; Dumbacher et al., 2012; McCormack et al., 2012). Within the Afrotheria are species that are fully aquatic (such as manatees and dugongs), extremely large (elephants), fossorial (golden moles), semi-aquatic (otter shrews), insectivorous (aardvarks, elephants shrews and tenrecs) and omnivorous (hyraxes). These species present with a range of body sizes, phenotypes, habitats and life histories, and are considered an ancient radiation of the Eutherian mammals. In this sense, the study of neural systems that are general quite conservative in their evolution (e.g. Dell et al., 2010) is of interest, as a record of the changes, or indeed lack of changes, may reflect the potential evolutionary plasticity/malleability of the mammalian brain.

The cholinergic, catecholaminergic and serotonergic systems, have been studied previously in two species belonging to the Afrotheria - the rock hyrax, Procavia capensis (Gravett et al., 2009) and the eastern rock elephant shrew, Elephantulus myurus (Pieters et al., 2010). While for the most part, these systems were similar to those reported in other mammals, several specific differences were noted. In the rock hyrax, the anterior nuclei of the dorsal thalamus were found to contain cholinergic neurons, there were cholinergic parvocellular neurons forming a shell around the typically laterodorsal tegmental and penduclopontine tegemental nuclei, and the locus coeruleus proper was observed to be made up of very few cells (Gravett et al., 2009). In contrast, cholinergic neurons were observed in the both the superior and inferior colliculi and the cochlear nuclei, and the catecholaminergic anterior hypothalamic group 
(A15d) was missing from the elephant shrew (Pieters et al., 2010). Observations of the orexinergic neurons in the rock hyrax showed a typically mammalian orexinergic system in this species apart from a dense innervation of the anterior nuclei of the dorsal thalamus (Gravett et al., 2011).

In the present study we expand the observations made on these systems in the Afrotheria by examining, using immunohistochemical means, the cholinergic, catecholaminergic, serotonergic and orexinergic systems in the brains of the giant otter shrew (Potomogale velox), the Hottentot golden mole (Amblysomus hottentotus) and the four-toed sengi (Petrodromus tetradactylus). The giant otter shrew is a semi-aquatic member of the Tenrecidae that is found in the swamps, streams and forest pools of central Africa. The hottentot golden mole is a small fossorial golden mole found in the Eastern Cape region of South Africa and inhabits a range of ecosystems from temperate to tropical dry forests, dry savanna, through to urban areas and introduced vegetations. The four-toed sengi is a large elephant shrew, found in tropical to subtropical moist montane forests as well as moist savanna throughout large areas of subSaharan Africa.

As far as the authors are aware, the systems described in the current study have not been examined in any of these three species. In addition to the prior studies of Afrotherian species, the data from the current study provides a broader base for comparison within the Afrotheria and across mammals in general that may help to elucidate the manner in which systems level changes occur across mammalian brains as features such as brain size, phenotype, life histories, phylogenetic relationships, and time since evolutionary divergence, change (Manger, 2005).

\section{Methods and Materials}

Brains from three Afrotherian species (Potamogale velox, Amblysomus hottentotus and Petrodromus tetradactylus) were used in the present study. Permits were obtained from the relevant wildlife authorities in the Democratic Republic of Congo and South Africa for the capture and euthanasia of the animals from their natural habitat. All animals were handled according to the guidelines of the University of the Witwatersrand Animal Ethics Committee. Each animal was weighed, anaesthetized and subsequently euthanized with weight appropriate 
doses of sodium pentobarbital (200mg sodium pentobarbital $/ \mathrm{kg}$, i.p.). In the current study a single $P$. velox (body mass $-540 \mathrm{~g}$; brain mass $-3.46 \mathrm{~g}$; an adult male), two A. hottentotus (body masses $-72 \mathrm{~g}, 86 \mathrm{~g}$; brain masses $-1.3 \mathrm{~g}, 1.2 \mathrm{~g}$; both adult males), and three $P$. tetradactylus (body masses - $132 \mathrm{~g}, 138 \mathrm{~g}, 124 \mathrm{~g}$; brain masses $-3.01 \mathrm{~g}, 2.80 \mathrm{~g}, 2.95 \mathrm{~g}$; all adult males) were used. Upon cessation of respiration the animals were perfused intracardially with $0.9 \%$ saline followed by $4 \%$ paraformaldehyde in $0.1 \%$ phosphate buffer (PB), approximately $11 / \mathrm{kg}$ of each solution, both solutions having a temperature of approximately $4^{\circ} \mathrm{C}$. The brains were then carefully removed from the skulls and post-fixed over night in $4 \%$ paraformaldehyde in $0.1 \mathrm{M}$ $\mathrm{PB}$ followed by equilibration in $30 \%$ sucrose in $0.1 \mathrm{M} \mathrm{PB}$. A one in six series of sections was used for Nissl, myelin, choline-acyltransferase (ChAT), tyrosine hydroxylase (TH), serotonin (5HT) and orexin (hypocretin/OxA). Sections used for the Nissl series were mounted on $0.5 \%$ gelatine-coated glass slides, cleared in a solution of 1:1 chloroform and absolute alcohol, then stained in $1 \%$ cresyl violet to reveal cell bodies. Myelin sections were first stored in $5 \%$ formalin for two weeks at $4^{\circ} \mathrm{C}$ then mounted on $1.5 \%$ gelatine-coated slides and subsequently stained with silver solution to reveal myelin sheaths (Gallyas, 1979).

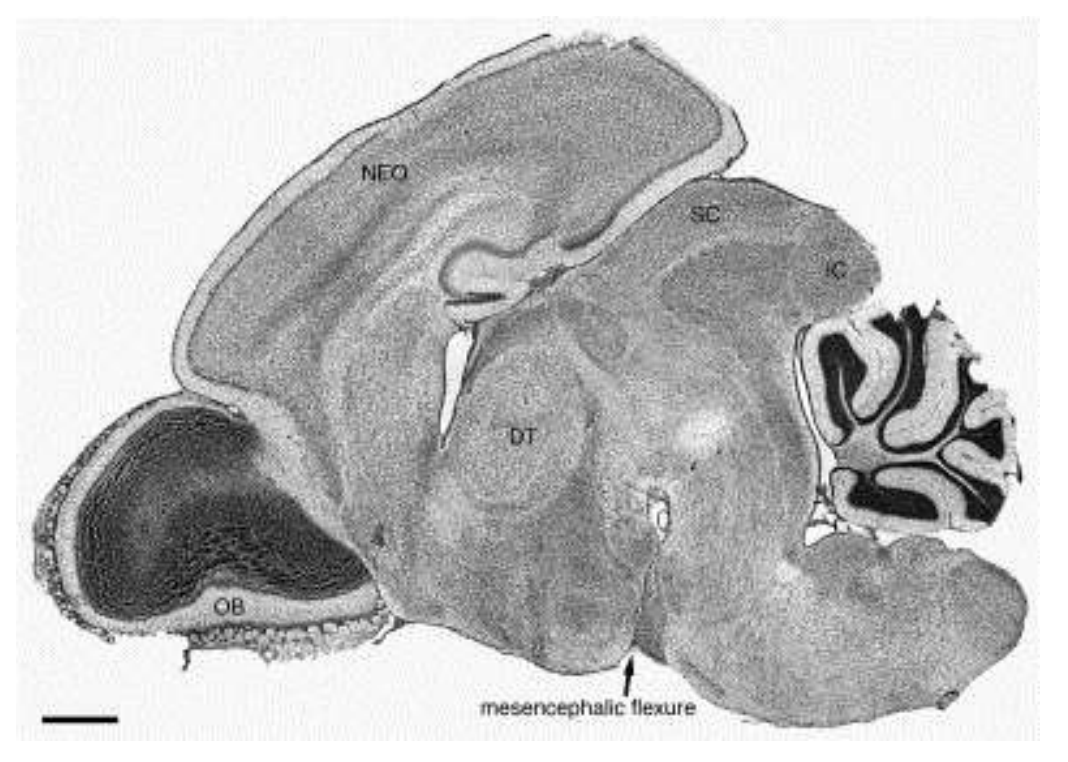

Figure 1: Photomontage of a Nissl-stained parasagittal section of the brain of the Hottentot golden mole showing the unusual appearance of the diencephalon and brainstem. It appears that a foreshortening and widening of the cranium leads to the induction/maintenance of a large flexure of the subtelencephalic brain, resulting in the pons lying beneath the midbrain, and the inferior colliculus lying above the cerebellum. Scale bar = $1 \mathrm{~mm}$. 
For the immunohistochemical staining each section was treated with endogenous peroxidase inhibitor (49.2\% 0.1M PB: $1.6 \%$ of $30 \% \mathrm{H}_{2} \mathrm{O}_{2}$ ) for $30 \mathrm{~min}$ and subsequently subjected to three $10 \mathrm{~min} 0.1 \mathrm{M}$ PB rinses. Sections were then preincubated for $2 \mathrm{~h}$, at room temperature, in blocking buffer (3\% normal goat serum, NGS, for TH, serotonin and orexin sections; 3\% normal rabbit serum, NRS, for ChAT sections, $2 \%$ bovine serum albumin for all sections and $0.25 \%$ Triton-X in $0.1 \mathrm{M}$ PB for all sections). This was followed by three $10 \mathrm{~min}$ rinses in $0.1 \mathrm{M} \mathrm{PB}$. The sections were then placed at $4^{\circ} \mathrm{C}$ under constant gentle shaking in primary antibody solution that contained the appropriate diluted primary antibody in blocking buffer (see above) for $48 \mathrm{~h}$. Anticholineacetyltransferase (AB144P, Millipore, raised in goat) at a dilution of 1:3000 was used to reveal cholinergic neurons. Anti-tyrosine hydroxylase (AB151, Millipore, raised in rabbit) at a dilution of 1:7500 revealed the putative catecholaminergic neurons. Serotonergic neurons were revealed using anti-serotonin (AB938, Millipore, raised in rabbit) at a dilution of 1:10000. Orexinergic neurons were revealed using anti-Orexin A (AB3704, Millipore, raised in rabbit) at a dilution of 1:3000. This incubation was followed by three $10 \mathrm{~min}$ rinses in $0.1 \mathrm{M} \mathrm{PB}$ and the sections were then incubated in a secondary antibody solution (1:1000 dilution of biotinylated anti-rabbit IgG, BA-1000, Vector Labs, for TH, serotonin and orexin sections, or a 1:1000 dilution of biotinylated anti-goat IgG, BA-5000, Vector Labs, for ChAT sections, in a blocking buffer containing $3 \% \mathrm{NGS} / \mathrm{NRS}$ and $2 \% \mathrm{BSA}$ in $0.1 \mathrm{M} \mathrm{PB}$ ) for $2 \mathrm{~h}$ at room temperature. This was followed by three $10 \mathrm{~min}$ rinses in $0.1 \mathrm{M} \mathrm{PB}$, after which sections were incubated for $1 \mathrm{~h}$ in Avidin-Biotin solution (Vector Labs), followed by three 10 min rinses in 0.1M PB. Sections were then placed in a solution of $0.05 \%$ diaminobenzidine (DAB) in $0.1 \mathrm{M}$ PB for $5 \mathrm{~min}$, followed by the addition of $3 \mu \mathrm{l}$ of $3 \%$ hydrogen peroxide to each $1 \mathrm{ml}$ of solution in which each section was immersed. Chromatic precipitation was visually monitored and verified under a low power stereomicroscope. Staining continued until such time as the background stain was at a level that would assist reconstruction without obscuring the immunopositive neurons. Precipitation was arrested by placing sections in $0.1 \mathrm{M} \mathrm{PB}$, followed by two more rinses in this solution. Sections were then mounted on $0.5 \%$ gelatine coated glass slides, dried overnight, dehydrated in a graded series of alcohols, cleared in xylene and coverslipped with Depex. The controls employed in this experiment included the omission of the primary antibody and the omission of the secondary antibody in selected sections for which no staining was evident.

Sections were examined under a low power stereomicroscope and using a camera lucida, 
the architectonic borders of the sections were traced following the Nissl and myelin stained sections. Sections containing the immunopositive neurons were matched to the drawings and the neurons were marked. All drawings were then scanned and redrawn using the Canvas 8 drawing program. The nomenclature used for the cholinergic system was adopted from Woolf (1991), Limacher et al. (2008), Bhagwandin et al. (2008), Gravett et al. (2009) and Pieters et al. (2010), the catecholaminergic from Hökfelt et al. (1984), Smeets and Gonzalez (2000), Limacher et al. (2008), Bhagwandin et al. (2008), Gravett et al. (2009) and Pieters et al. (2010), the serotonergic from Törk (1990), Limacher et al. (2008), Bhagwandin et al. (2008), Gravett et al. (2009) and Pieters et al. (2010), and the orexinergic from Kruger et al. (2010), Bhagwandin et al. (2011) and Gravett et al. (2011). While we use the standard nomenclature for the catecholaminergic system in this paper, we realise that the neuronal groups revealed with tyrosine hydroxylase immunohistochemistry may not directly correspond with these nuclei as has been described in previous studies by Dahlström and Fuxe (1964), Hökfelt et al. (1976), Meister et al. (1988), Kitahama et al. (1990, 1996), and Ruggiero et al. (1992); however, given the striking similarity of the results of the tyrosine hydroxylase immunohistochemistry to that seen in other mammals, we feel this terminology is appropriate. Clearly further studies in these Afrotherian species with a wider range of antibodies, such as those to phenylethanolamine-N-methyltransferase (PNMT), dopamine- $\beta$-hydroxylase (DBH) and aromatic L-amino acid decarboxylase (AADC) would be required to fully determine the implied homologies ascribed in this study (e.g. Weihe et al., 2006). We address this potential problem with the caveat of putative catecholaminergic neurons where appropriate in the text.

\section{Results}

The current study describes and defines the nuclear organization of the cholinergic, catecholaminergic, serotonergic and orexinergic neural systems in three species of Afrotherian mammals (giant otter shrew - Potomogale velox, the Hottentot golden mole - Amblysomus hottentotus, and the four-toed sengi - Petrodromus tetradactylus) in which these systems have not been previously described. For the most part, the systems investigated exhibited an organization that may be thought of as typically mammalian; however, we did observe many points of departure from this typical organization. One central observation of interest in the current study, and of importance in the interpretation of the organization of the systems studied, 

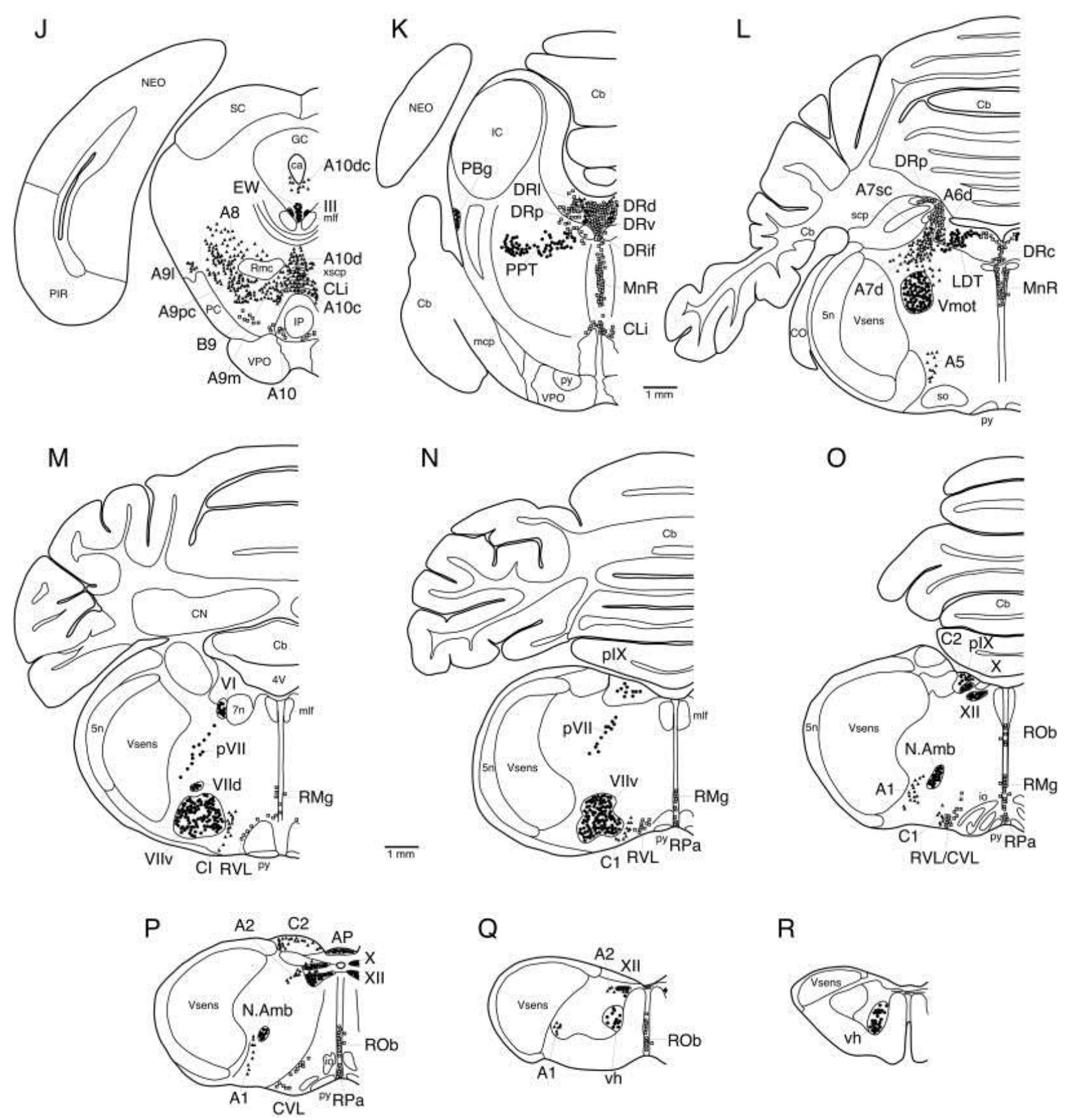

Figure 2: Serial drawings of coronal sections through one half of the giant otter shrew (Potomogale velox) brain from the olfactory bulb through to the spinomedullary junction. $\mathbf{A}$ is the most rostral section, $\mathbf{R}$ the most caudal. The outlines of the architectonic regions were drawn using Nissl and myelin stains and immunoreactive cells marked on the drawings. Solid black circles depict cholinergic neurons, solid triangles depict catecholaminergic neurons (those immunoreactive for tyrosine hydroxylase), open squares depict serotonergic neurons and closed stars represent orexinergic neurons. Each circle, triangle, square or star represents an individual neuron. The figures are approximately $1500 \mu \mathrm{m}$ apart. See list for abbreviations. 
was the noticeable folding, or compression, of the brainstem of the golden mole. Within the brain of the golden mole, the axial alignment of the brainstem was foreshortened in such a manner that the pontine region was observed to lie beneath the midbrain, and not caudal to it as seen in most other mammals (Fig. 2).

\subsection{Cholinergic nuclei}

The cholinergic system is generally subdivided into six main regions containing a cluster of distinct nuclei: cortical interneurons; striatal; basal forebrain; diencephalic; pontomesencephalic; and cranial motor nerve nuclei (Woolf, 1991). Cholineacetyltransferase immunoreactive neurons (ChAT+) were identified in all these subdivisions, except the cortical interneurons, which were only identified in the Hottentot golden mole (Figs. 2, 3, 4, 5). In addition to the cortical cholinergic interneurons, the golden mole also evinced cholinergic interneurons in the olfactory bulb, piriform cortex, amygdala and hippocampus (Fig. 5). Interestingly, the four-toed sengi possessed cholinergic interneurons in both superior and inferior colliculi and the cochlear nuclear complex (Fig. 4L-P), as described for the eastern rock elephant shrew (Pieters et al., 2010).

\subsubsection{Cholinergicinterneurons in the telencephalon of the golden mole}

Previous studies have shown that while cholinergic interneurons are observed in the cerebral cortex of certain species, these are most readily observed in rodents of the Murid family (Bhagwandin et al., 2006; Kruger et al., 2012). Within the Hottentot golden mole, there was an abundance of typically bipolar cholinergic neurons (Fig. 3A-K), oriented orthogonal to the pial surface of the cerebral cortex, throughout all layers of the cortex except layer 1. Similar cholinergic interneurons were also observed throughout the piriform cortex. Interestingly, this species also had cholinergic interneurons in the glomerular, inner granular and periventricular layers of the olfactory bulb, as well as in the CA region of the hippocampus and throughout the amygdala nuclear complex (Fig. 5). Similar cholinergic neurons were not observed in the brains of the giant otter shrew or the four-toed sengi (Figs. 2, 4). 

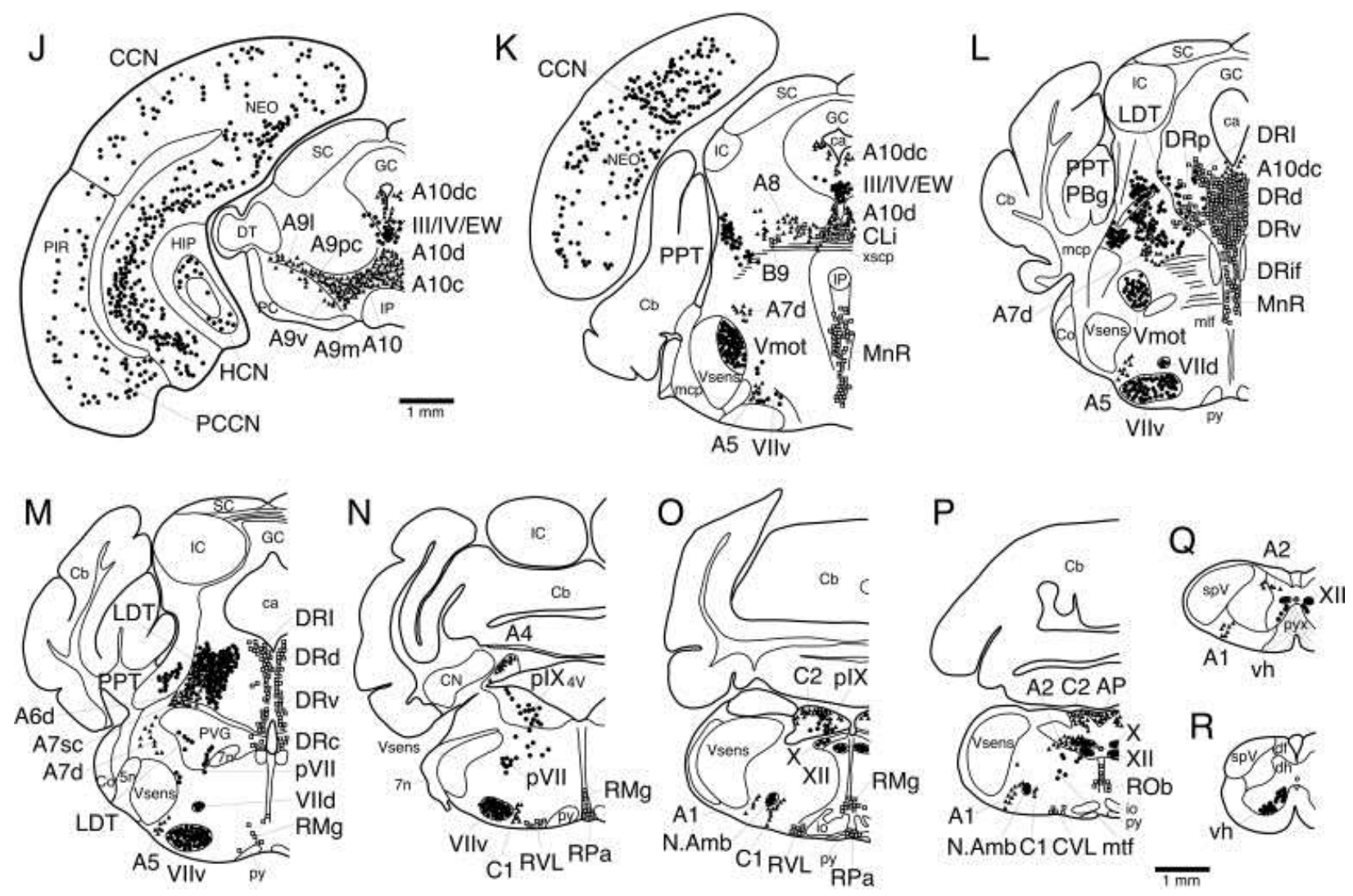

Figure 3: Serial drawings of coronal sections through one half of the Hottentot golden mole (Amblysomus hottentotus) brain from the olfactory bulb through to the spinomedullary junction. $\mathbf{A}$ is the most rostral section, $\mathbf{R}$ the most caudal. The outlines of the architectonic regions were drawn using Nissl and myelin stains and immunoreactive cells marked on the drawings. Solid black circles depict cholinergic neurons, solid triangles depict catecholaminergic neurons (those immunoreactive for tyrosine hydroxylase), and open squares depict serotonergic neurons. Each circle, triangle or square represents an individual neuron. The figures are approximately $750 \mu \mathrm{m}$ apart. See list for abbreviations. 
A

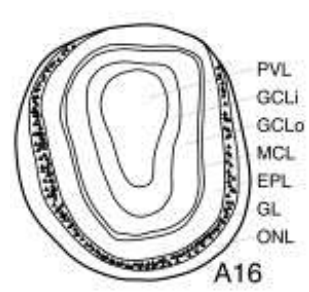

B

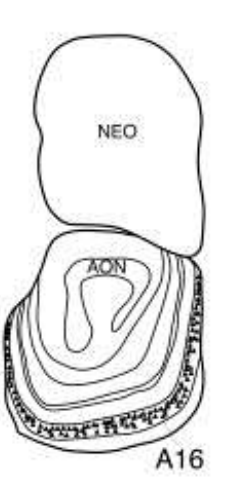

C

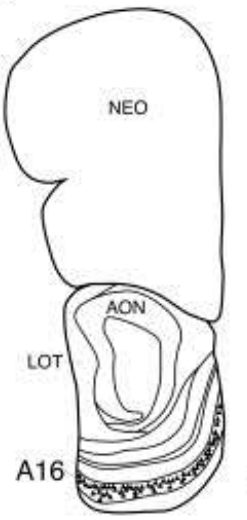

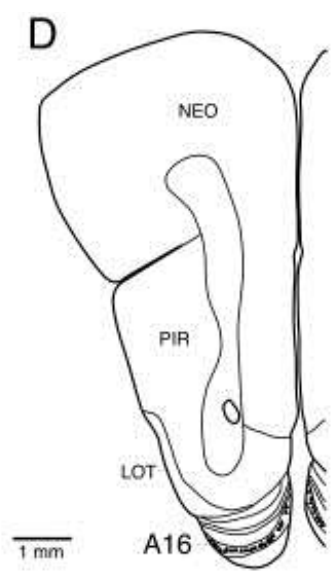
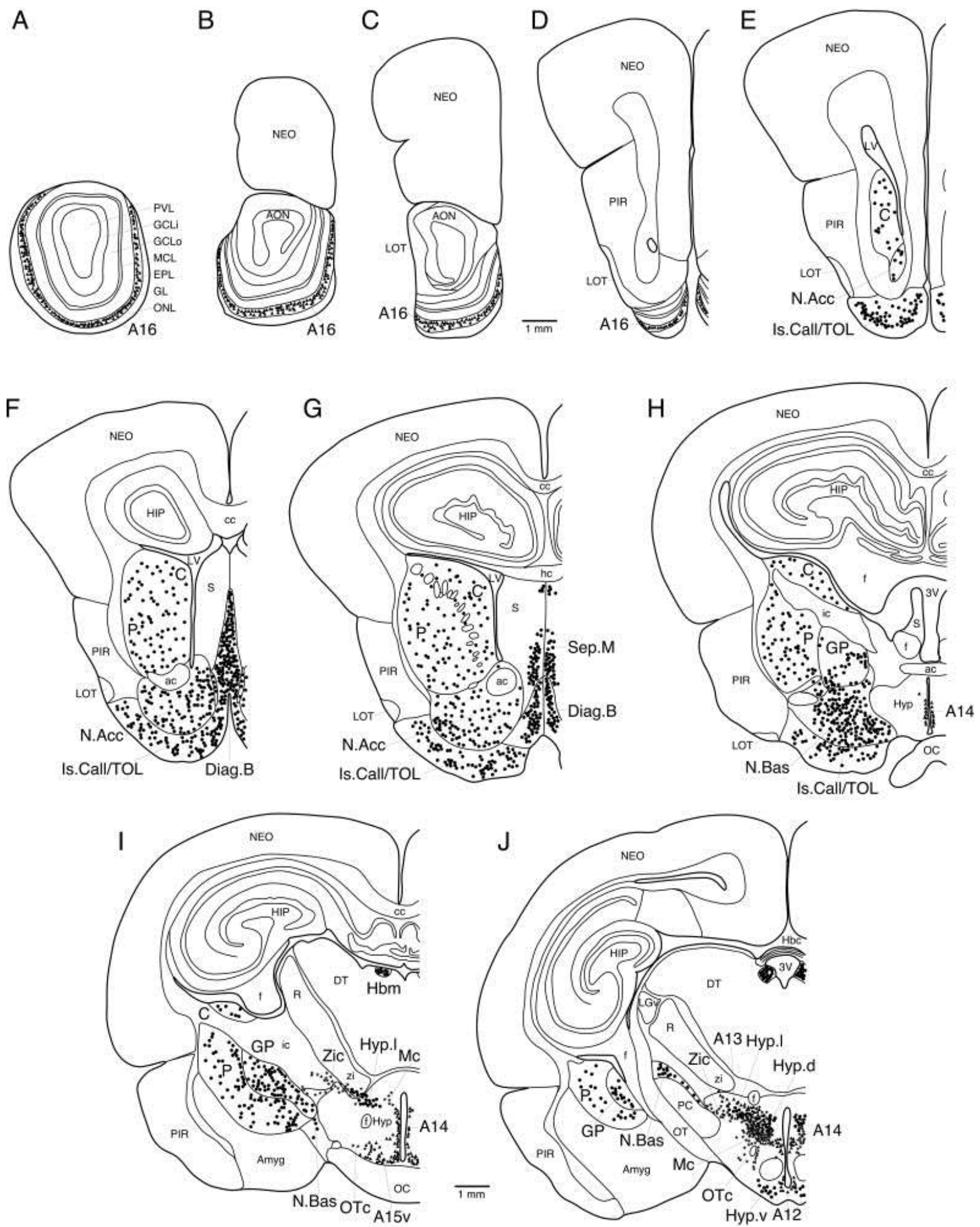

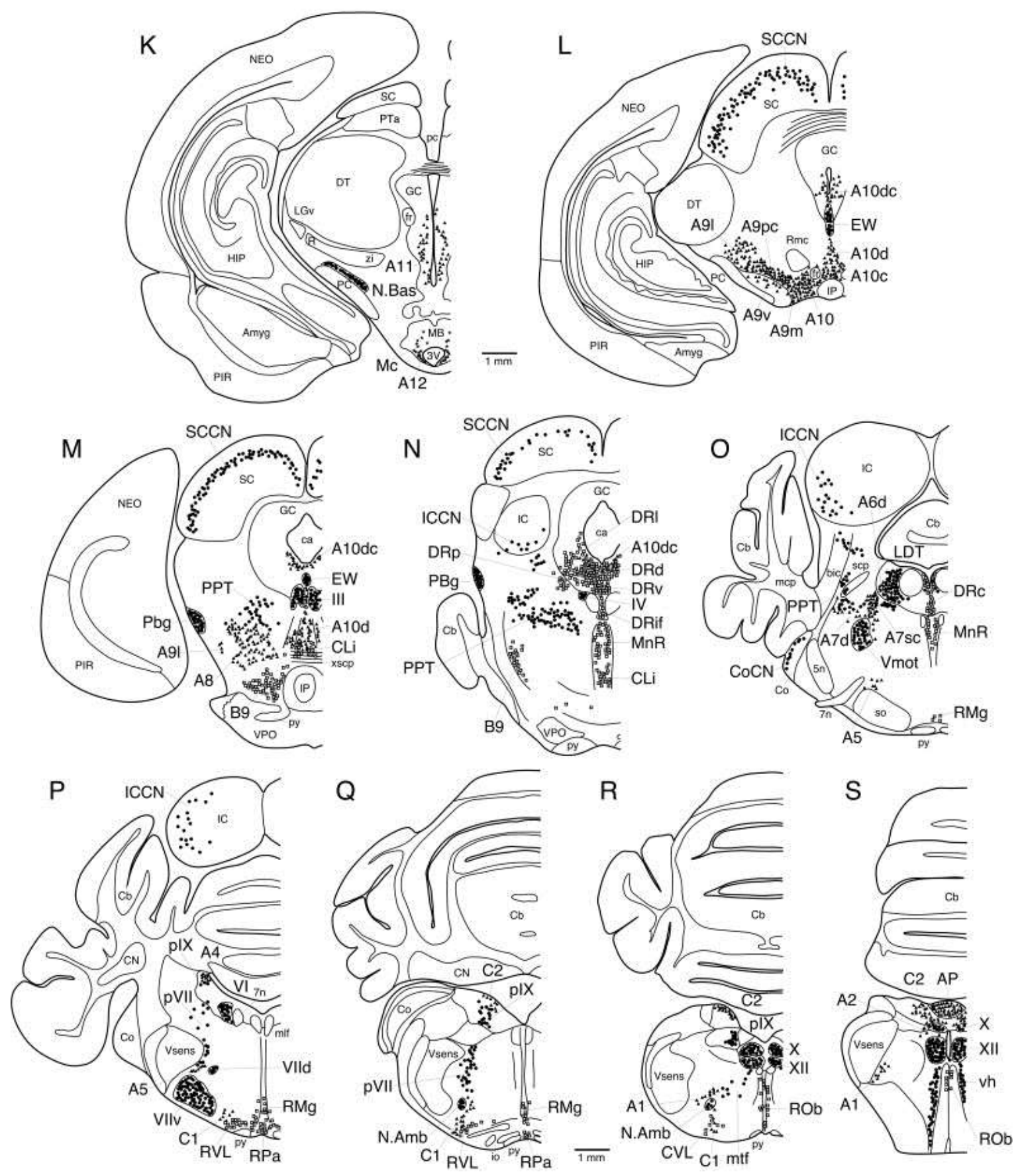

Figure 4: Serial drawings of coronal sections through one half of the four-toed sengi (Petrodromus tetradactylus) brain from the olfactory bulb through to the spinomedullary junction. $\mathbf{A}$ is the most rostral section, $\mathbf{S}$ the most caudal. The outlines of the architectonic regions were drawn using Nissl and myelin stains and immunoreactive cells marked on the drawings. Solid black circles depict cholinergic neurons, solid triangles depict catecholaminergic neurons (those immunoreactive for tyrosine hydroxylase), open squares depict serotonergic neurons and closed stars represent orexinergic neurons. Each circle, triangle, square or star represents an individual neuron. The figures are approximately $1200 \mu \mathrm{m}$ apart. See list for abbreviations. 

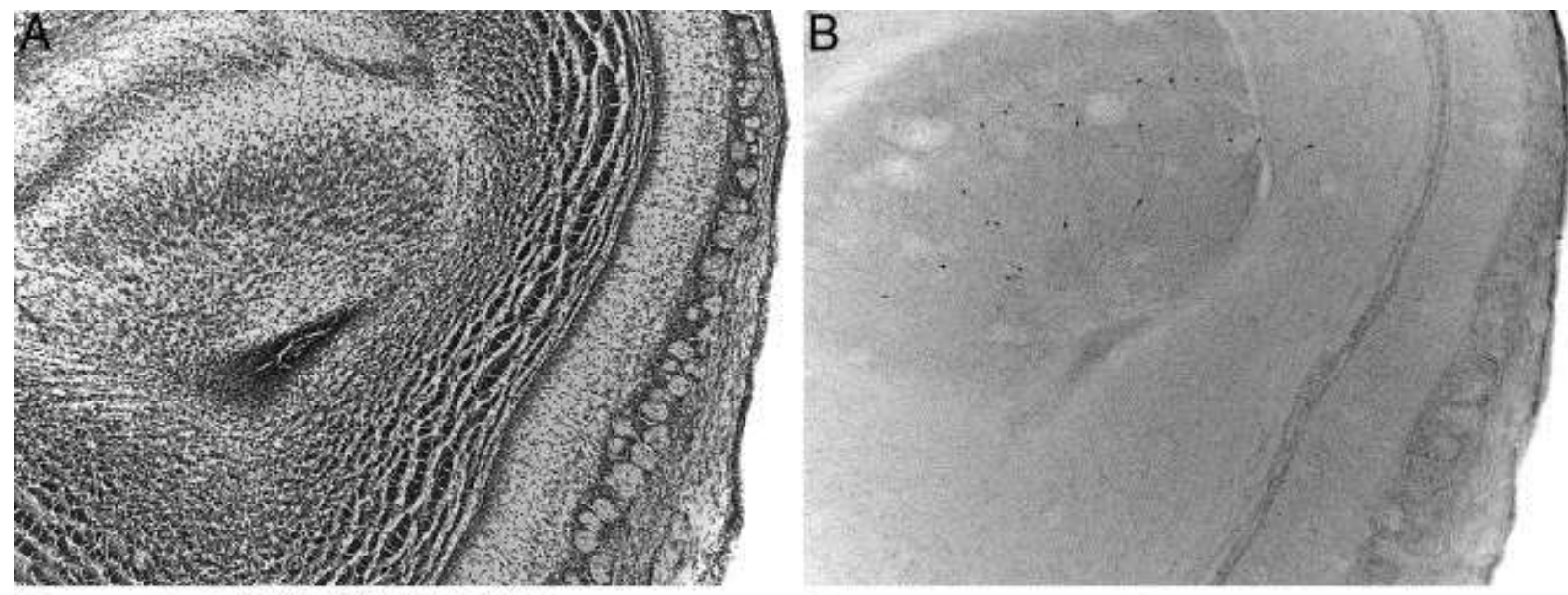

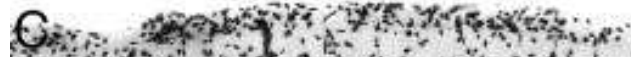

The

D

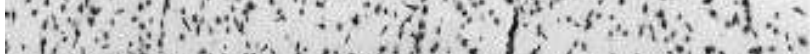

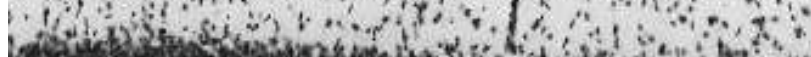
63 10.

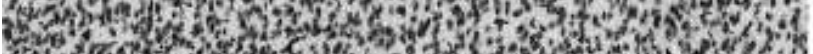

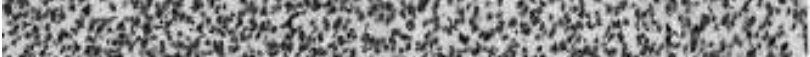

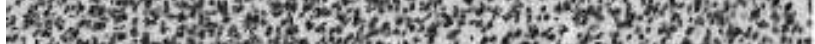

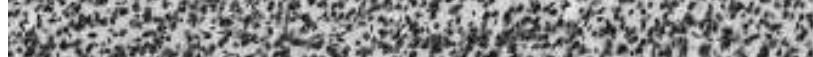

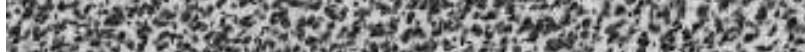

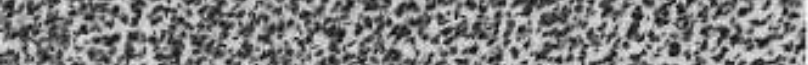

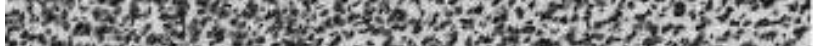

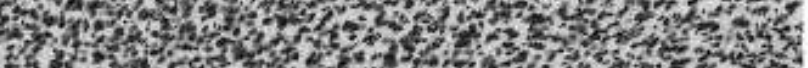

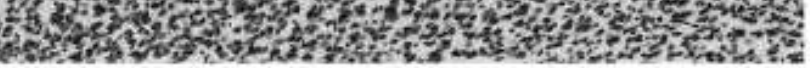
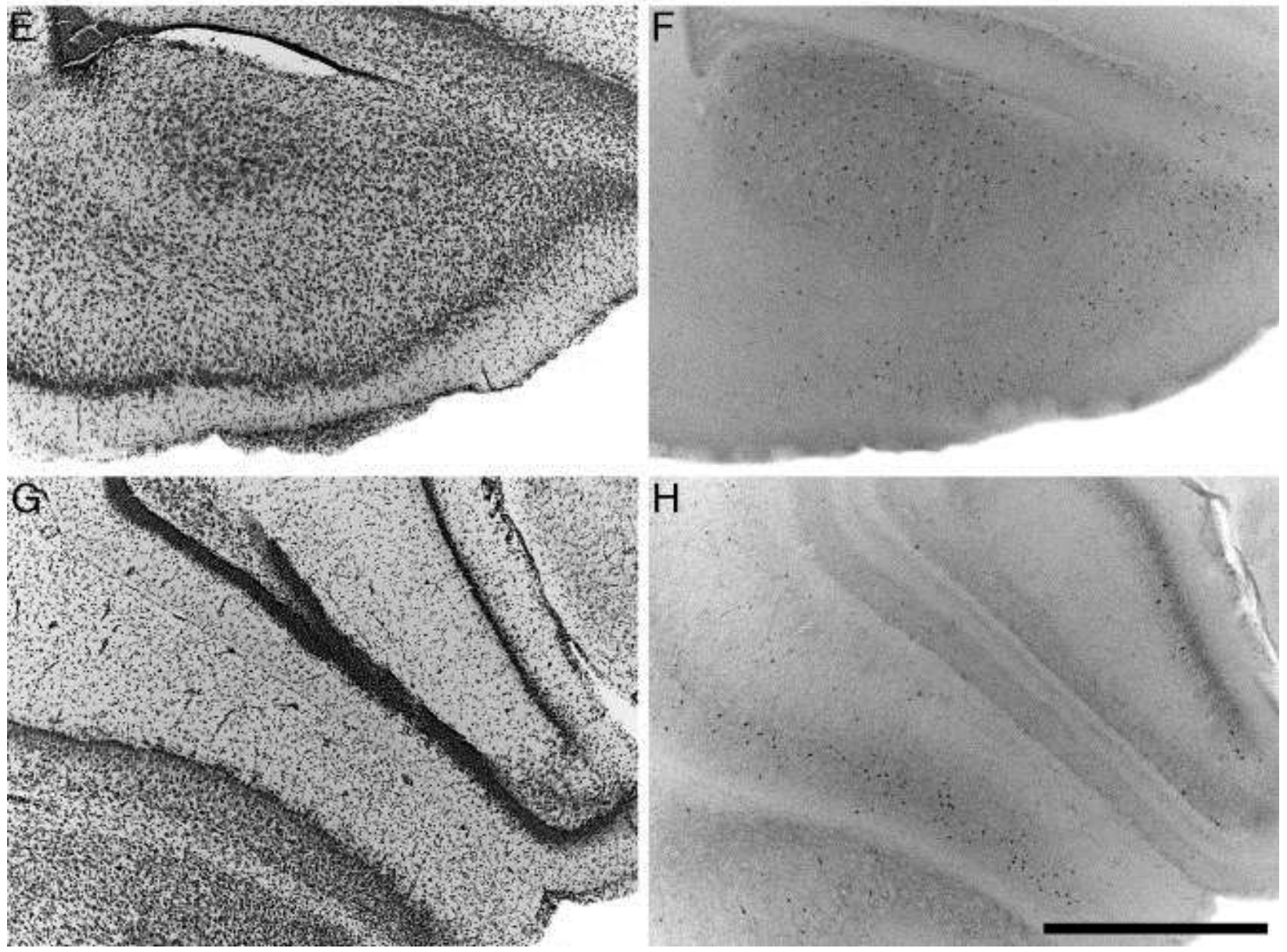
Figure 5: Photomicrographs of sections stained for Nissl substance (A, C, E, G) and immunoreactivity for cholineacetyltransferase $(\mathbf{B}, \mathbf{D}, \mathbf{F}, \mathbf{H})$, showing the location and appearance of cholinergic interneurons in the brain of the Hottentot golden mole. A and $\mathbf{B}$ are taken through the olfactory bulb, showing the cholinergic interneurons in the periventricular cell layer. $\mathbf{C}$ and $\mathbf{D}$ are of the cerebral neocortex showing the cholinergic interneurons in the upper layers of the cortex. $\mathbf{E}$ and $\mathbf{F}$ are taken through the centre of the amygdaloid complex showing the cholinergic interneurons located throughout this nuclear mass. $\mathbf{G}$ and $\mathbf{H}$ are taken through the hippocampal formation, showing the presence of cholinergic interneurons in the cornu ammonis and dentate gyrus. The scale bar in $\mathbf{D}=500 \mu \mathrm{m}$ and applies to $\mathbf{C}$ and $\mathbf{D}$, the scale bar in $\mathbf{H}=1000 \mu \mathrm{m}$, and applies to $\mathbf{A}, \mathbf{B}, \mathbf{E}, \mathbf{F}, \mathbf{G}$ and $\mathbf{H}$.

\subsubsection{Striatal cholinergic interneurons}

ChAT+ neurons were found in the caudate/putamen complex, the globus pallidus, the nucleus accumbens, the Islands of Calleja and the olfactory tubercle in all three species (Figs. 2C-G, 3D-G, 4E-J). A moderate density of ChAT+ neurons was found within the caudate/putamen and throughout the globus pallidus, but most densely at its borders with the putamen and nucleus basalis. Through the nucleus accumbens a moderate density of ChAT+ neurons was observed, and at the ventral border of this nucleus, they appear to intermingle with the most dorsal cholinergic neurons of the olfactory tubercle. The ChAT+ neurons within the olfactory tubercle and Islands of Calleja were found in the most ventral portion of the anterior telencephalon. Throughout the olfactory tubercle a moderate density of ChAT+ neurons were observed, and within the most ventral portion of this region clusters of ChAT+ neurons were observed to form the Islands of Calleja. The clustering of the ChAT+ neurons forming the Islands of Calleja was most pronounced in the Hottentot golden mole (Fig. 6A, 6B). 

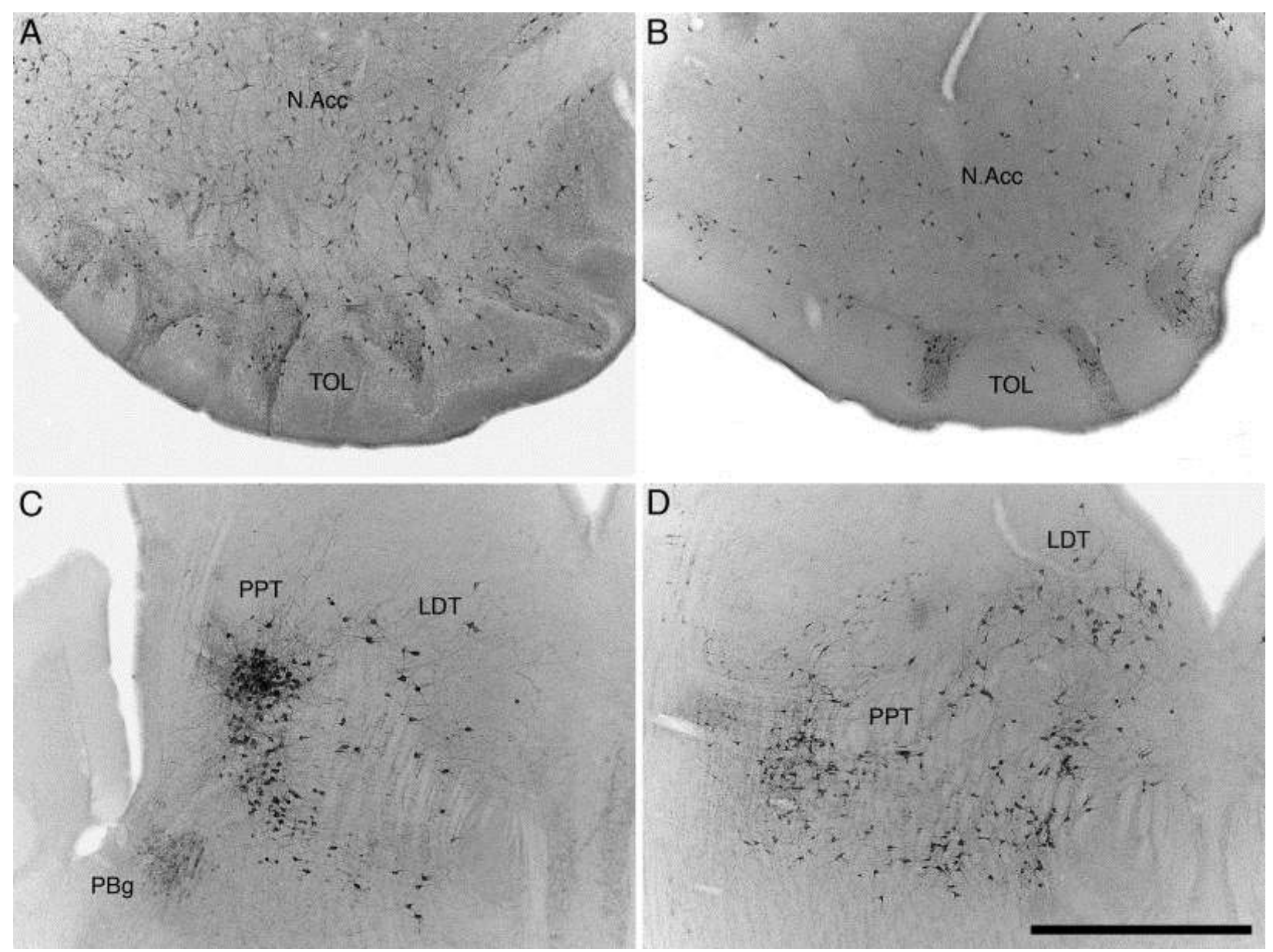

Figure 6: Photomicrographs showing neuronal groups immunoreactive for choline acetyltransferase in the olfactory tubercle (TOL) and nucleus accumbens (N.Acc) (A and B) and pontine region $(\mathbf{C}$ and $\mathbf{D})$ of the Hottentot golden mole (A and $\mathbf{C}$ ) and the four-toed sengi (B and $\mathbf{D})$. Note the elaborated appearance of the TOL in the Hottentot golden mole (A) in comparison to the same region in the four-toed sengi $(\mathbf{B})$ and the standard mammalian appearance and location of the laterodorsal tegmental (LDT), pedunculopontine tegmental (PPT) and parabigeminal (PBg) nuclei in both species ( $\mathbf{C}$ and $\mathbf{D})$. Scale bar in $\mathbf{D}=1000 \mu \mathrm{m}$ and applies to all.

\subsubsection{Cholinergic nuclei of the basal forebrain}

Cholinergic nuclei that could be identified within the basal forebrain of the three Afrotherian species studied included the medial septal nucleus, the Diagonal band of Broca and the nucleus basalis (Figs. 2D-I, 3E-I, 4F-K). The medial septal nucleus exhibited a moderate to high density of ChAT+ neurons and was located within the rostral half of the medial wall of the septal nuclear complex immediately below the rostrum of the corpus callosum. In the Hottentot 
golden mole, a distinct cluster of ChAT+ neurons was observed in the lateral septal nucleus. These lateral septal cholinergic neurons exhibited a similar morphology to those observed in the medial septal nucleus of this species. The ChAT + neurons forming the diagonal band of Broca were evidenced as a high density of neurons located in the ventromedial corner of the cerebral hemisphere anterior to the hypothalamus. It was possible to divide this nucleus into both horizontal and vertical limbs, but this was not deemed necessary since it would not add any value to the description. A cluster of moderate to high-density ChAT+ neurons located anterior and ventral to the globus pallidus and caudal to the olfactory tubercle were assigned to the nucleus basalis. The ChAT+ neurons of this nucleus appear to be continuous with those of the globus pallidus in all three species, and interestingly, this nucleus appears to extend quite caudally in all three species, forming a small cluster that finally terminates above the lateral margin of the cerebral peduncle at the level of the mammillary bodies.

\subsubsection{Diencephalic cholinergic nuclei}

In all three species ChAT+ neurons were found in the medial habenular nucleus, as well as the dorsal, lateral and ventral hypothalamic clusters (Figs. 2F-G, 3G-H, 4I-J). The medial habenular nucleus was located in the dorsomedial aspect of the diencephalon adjacent to the third ventricle and the ChAT+ neurons within this nucleus were very densely packed. The three clusters of ChAT+ neurons within the hypothalamus all showed moderate to weak immunoreactivity, but were clearly observed. The dorsal cluster was found in the dorsomedial aspect of the hypothalamus between the third ventricle and fornix, the lateral cluster was found in the dorsolateral aspect of the hypothalamus, lateral to the fornix, while the ventral cluster was located in the ventral medial portion of the hypothalamus adjacent to the neurons of the catecholaminergic A12 nucleus (see below).

\subsubsection{Pontomesencephalic cholinergic nuclei}

ChAT+ immunoreactive neurons were delineated the parabigeminal nucleus, and the pedunculopontine (PPT) and laterodorsal (LDT) nuclei in all three species investigated (Figs. 
2K-L, 3K-M, 4M-O, 6C, 6D). The parabigeminal nucleus was located at the very lateral margin of the pontine tegmentum in a location ventral to the inferior colliculus. Within this nucleus the ChAT+ neurons were moderately densely packed, although in both the giant otter shrew and the Hottentot golden mole, this nucleus contained less neurons that the same nucleus in the four-toed sengi. A moderate to high density of ChAT+ neurons forming the PPT were located within the dorsal aspect of the pontine tegmentum surrounding the superior cerebellar peduncle, even in the unusually arranged brainstem of the Hottentot golden mole. Within the periventricular grey matter, caudal to the oculomotor nucleus (inferior to this nucleus in the Hottentot golden mole), a moderate to high density of ChAT+ neurons were designated as those forming the LDT nucleus. The ventrolateral border of the LDT neurons was contiguous with the dorsomedial border of the PPT nucleus, the only reason to separate these two nuclei being the transition from the periventricular grey matter to the pontine tegmentum.

\subsubsection{Collicular and Cochlear cholinergic interneurons of the four-toed sengi}

Within the brain of the four-toed sengi ChAT+ neurons were observed in the superior and inferior colliculi, and the cochlear nucleus (Fig. 4L-O). These neurons were not observed in either the giant otter shrew or the Hottentot golden mole. These ChAT+ neurons observed in the superior colliculus were found in the superficial layers, in a moderate to low density, were bipolar, with the dendrites oriented orthogonal to the pial surface. A low density of bipolar neurons of similar appearance was observed in the ventrolateral portion of the inferior colliculus; however, they exhibited no specific dendritic orientation. A few ChAT+ neurons were observed in the superficial layers of the cochlear nucleus and these were observed to be a mixture of bipolar and multipolar types.

\subsubsection{Cholinergic cranial nerve motor nuclei}

These nuclei were found in positions typical of all mammals (Woolf, 1991; Manger et al., 2002a; Maseko et al., 2007; Dell et al., 2010; Kruger et al, 2010). The ChAT+ nucleiidentified in the three Afrotherian species studied include: the oculomotor (III), trochlear (IV), motor division 
of thetrigeminal (Vmot), adbucens (VI), dorsal and ventral subdivisionsof the facial (VIId and VIIv), nucleus ambiguus, dorsal motor vagus(X), hypoglossal (XII), Edinger-Westphal (EW), medullary tegmentalfield (mtf) andthe preganglionic motor neurons of thesalivatory (pVII) and the glossopharyngeal (pIX) nerves (Figs. 2J-R, 3J-R, 4L-S). There were certain species specific aspects of these nuclei including the lack of a medullary tegmental field and a very small trochlear nucleus in the giant otter shrew, and a small number of neurons that combined form the oculomotor, trochlear and Edinger-Westphal nuclei in the Hottentot golden mole.

\subsection{Putative catecholaminergic nuclei}

Tyrosine hydroxylase $(\mathrm{TH}+)$ immunoreactivity revealed the putatively catecholaminergic neurons in the brains of the three species examined. The nuclei formed by these neurons were arranged in a number of identifiable nuclear complexes that extended from the olfactory bulb through to the spinomedullary junction. These complexes correspond to that seen in other mammals (e.g. Smeet and Gonzalez, 2000) and could be divided into the olfactory bulb, diencephalic, midbrain, pontine and medullary nuclear clusters. In the current description the nuclei are referred to using the nomenclature of Dahlström and Fuxe (1964) and Hökfelt et al. (1984), as no putatively catecholaminergic nuclei outside the classically defined nuclei (e.g. Smeet and Gonzalez, 2000) were observed. The TH+ nuclei found in the three species studied were similar to that seen in many other mammals, but there were some exceptions. The rodent typical C3 nucleus (rostral dorsal midline medullary nucleus) and the Murid rodent/primate/megabat A6c nucleus (compact portion of locus coeruleus) (e.g. Dell et al., 2010; Kruger et al, 2012) were absent in all three species. The A15d nucleus (anterior hypothalamic group, dorsal division) was absent in the four-toed sengi, as seen for the eastern rock elephant shrew (Pieters et al., 2010), and the A6d nucleus (diffuse portion of locus coeruleus) was comparatively small in both the Hottentot golden mole and the four-toed sengi.

\subsubsection{Olfactory bulb (A16)}

The neurons forming the A16 nucleus were observed to be dense clusters of TH+ cells in the glomerular layer of the olfactory bulb in all three species (Figs. 2A, 3A-C, 4A-D). These 
neurons likely represent the periglomerular dopaminergic interneurons, were small in size, and were found in equal density surrounding the entire glomeruli.

\subsubsection{Diencephalic nuclei $($ A15 - A11)}

In the hypothalamus of all three species $\mathrm{TH}+$ cells formed six distinct nuclei: the dorsal division of the anterior hypothalamic group (A15d - except in the four-toed sengi, where this nucleus was absent), the ventral division of the anterior hypothalamic group (A15v), the rostral periventricular cell group (A14), the zona incerta (A13), the tuberal cell group (A12), and the caudal diencephalic group (A11) (Figs. 2F-I, 3G-I, 4H-K, 7A-C). Within the dorsal anterior portion of the hypothalamus of the Hottentot golden mole and giant otter shrew, between the third ventricle and the fornix, a moderate density of TH+ neurons was designated as the A15d nucleus. The $\mathrm{TH}+$ neurons forming the $\mathrm{A} 15 \mathrm{v}$ nucleus were located in the ventrolateral portion of the hypothalamus in a moderate density close to the floor of the brain and in the four-toed sengi these neurons appeared to be more numerous than in the other two species examined. A low to moderate density of $\mathrm{TH}+$ neurons forming two columns adjacent to the lateral walls of the third ventricle, were assigned to the A14 nucleus. Within the dorsolateral aspect of the hypothalamus, lateral to the fornix and intermingling with the neurons forming the zona incerta of the ventral thalamus, was a moderate density of $\mathrm{TH}+$ neurons forming to the A13 nucleus. $\mathrm{TH}+$ neurons assigned to the A12 nucleus were located in the ventromedial portion of the hypothalamus, surrounding and below the floor of the third ventricle in the arcuate nucleus and the immediate vicinity. In the four-toed sengi, these neurons extended into the regions around the mammillary bodies. Lastly, within the hypothalamic grey matter adjacent to the posterior pole of the third ventricle, a moderate density of $\mathrm{TH}+$ neurons formed the A11 nucleus, although the cells were less numerous in the giant otter shrew than in the other species. 

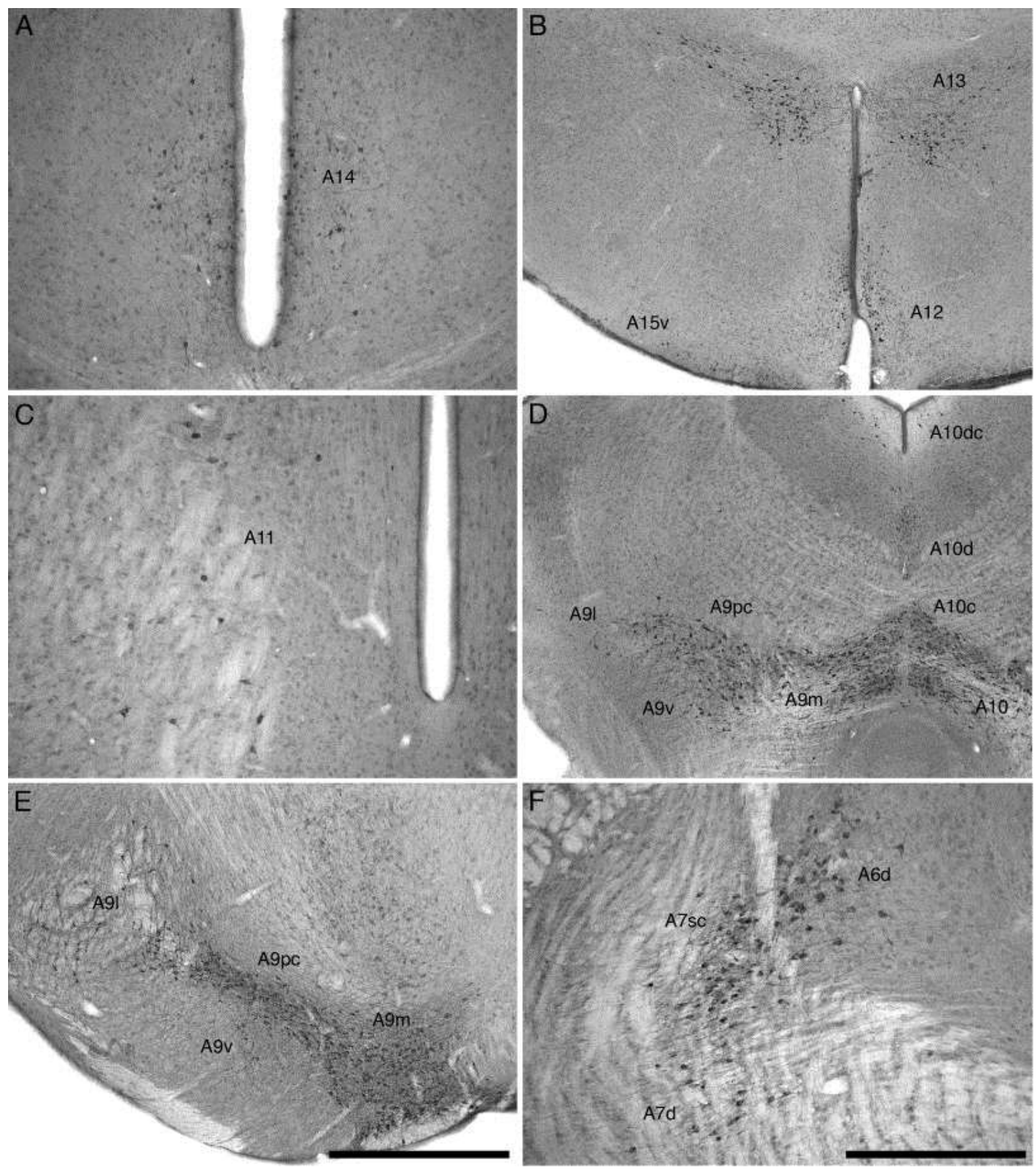

Figure 7: Photomicrographs showing neuronal groups that were immunopositive for tyrosine hydroxylase in the brains of the Afrotherians studied. A. The $\mathbf{A 1 4}$ cell group in the rostral hypothalamus of the giant otter shrew. B. The A15v, A13 and A12 cell groups in the middle hypothalamus of the Hottentot golden mole. C. The A11 groups in the caudal hypothalamus of the giant otter shrew. D. The ventral tegmental A10 complex and substantia nigra A9 complex in the midbrain of the Hottentot golden mole. E. The substantia nigra A9 complex in the midbrain of the four-toed sengi. $\mathbf{F}$. The locus coeruleus complex in the pontine region of the four-toed sengi. Scale bar in $\mathbf{E}=1000$ $\mu \mathrm{m}$ and applies to $\mathbf{B}, \mathbf{D}$ and $\mathbf{E}$. Scale bar in $\mathbf{F}=500 \mu \mathrm{m}$ and applies to $\mathbf{A}, \mathbf{C}$ and $\mathbf{F}$. See list for abbreviations. 


\subsubsection{Midbrain nuclei $(A 10-A 8)$}

Tyrosine hydroxylase immunoreactive neurons in the midbrain were found within the ventral tegmental area (the A10 complex, including the A10, A10c, A10d, A10dc nuclei), the substantia nigra (the A9 complex, including the A9pc, A91, A9v, A9m nuclei) and the retrorubral nucleus (A8) within the midbrain tegmentum in all three species studied (Figs. 2I-J, 3J-L, 4L-N, 7D-E). A high density of TH+ neurons, found dorsal and dorsolateral to the interpeduncular nucleus, between this nucleus and the root of the oculomotor nerve (IIIn), was assigned to the A10 nucleus. Immediately dorsal to the interpeduncular nucleus, in a location just anterior to the decussation of the superior cerebellar peduncle, a dense cluster of TH+ neurons formed the A10c nucleus. Dorsal to A10c, between it and the oculomotor nucleus, was a dense bilateral parasagittal cluster of $\mathrm{TH}+$ neurons that formed the A10d nucleus. The $\mathrm{TH}+$ neurons assigned to the A10dc nuclear complex were found within the periaqueductal grey matter surrounding the ventral half of the cerebral aqueduct.

The substantia nigra nuclear complex was observed in the ventral and lateral portions of the midbrain tegmentum, just dorsal to the cerebral peduncles. A9pc (pars compacta) was formed by a moderately dense band of $\mathrm{TH}+$ neurons that ran from medial to lateral immediately ventral to the medial lemniscus. Throughout the grey matter (pars reticulata of the substantia nigra) ventral to $\mathrm{A} 9 \mathrm{pc}$, scattered $\mathrm{TH}+$ neurons were assigned to the $\mathrm{A} 9 \mathrm{v}$ (ventral) nucleus. At the lateral edge of A9pc, a loose aggregation of $\mathrm{TH}+$ neurons formed the A9l (lateral) nucleus. Medial to A9pc and lateral to the root of the oculomotor nerve (IIIn), a dense cluster of $\mathrm{TH}+$ neurons formed the A9m (medial) nucleus. Scattered throughout the midbrain tegmentum, in a position caudal to the magnocellular division of the red nucleus and dorsal to the A9 complex, a sparsely packed but relatively numerous, cluster of $\mathrm{TH}+$ neurons that formed the A8 nucleus.

\subsubsection{Rostral rhombencephalon - the locus coeruleus complex, $A 7-A 4$}

Within the pontine region of all three species studied a large number of $\mathrm{TH}+$ neurons forming the locus coeruleus complex were readily observed. The locus coeruleus complex could be subdivided into five nuclei, these being: the subcoeruleus compact portion (A7sc), subcoeruleus diffuse portion (A7d), locus coeruleus compact portion (A6c), fifth arcuate nucleus (A5), and the dorsolateral division of locus coeruleus (A4) (Figs. 2L, 3K-N, 4O-P, 7F). Within 
the dorsal portion of the pontine tegmentum adjacent to the ventrolateral region of the periaqueductal grey matter, a tightly packed cluster of $\mathrm{TH}+$ neurons represented the $\mathrm{A} 7$ compact portion of the LC. This division is the same as what was previously described as the subcoeruleus (Dahlström and Fuxe, 1964; Olson and Fuxe, 1972). Ventral and lateral to the A7sc, a diffusely organised aggregation of $\mathrm{TH}+$ neurons formed the A7d nuclear complex. These neurons are located both medially and laterally around the trigeminal motor nucleus (Vmot). Within the lateral portion of the periventricular grey matter a loosely packed, moderate density of a moderate number of $\mathrm{TH}+$ neurons were assigned to the A6d nucleus in the giant otter shrew. In the Hottentot golden mole and the four-toed sengi, a lower number of less densely packed neurons were assigned as the A6d nucleus in these species. No compact division of the locus coeruleus (the A6c division as observed in rodents, e.g. Kruger et al., 2012) was observed in any of the three species examined. In the ventrolateral pontine tegmentum lateral to the superior olivary nucleus and ventrolateral to Vmot and A7d, a small cluster of TH+neurons formed the A5 nucleus. These neurons formed a rough meshlikedendritic network around the ascending fascicles located within the ventrolateral pontine tegmentum. Immediately adjacent to the wall of the fourth ventricle, in the dorsolateral portion of the periaqueductal grey matter, a very dense, but small cluster of $\mathrm{TH}+$ neurons represents the A4 nucleus.

\subsubsection{Medullary nuclei (C1, C2, A1, A2, Area postrema)}

In the medulla of all three species five putative catecholaminergic nuclei were observed: the rostral ventrolateral tegmental group $(\mathrm{C} 1)$, the rostral dorsomedial group $(\mathrm{C} 2)$, the caudal ventrolateral tegmental group (A1), the caudal dorsomedial group (A2), and the area postrema (AP) (Figs. 2M-Q, 3N-Q, 4P-S). A low density of TH+neurons, found in the ventrolateral medulla from the level of the facial nerve nucleus to the mid-level of nucleus ambiguus, were classified as the $\mathrm{C} 1$ nucleus. Continuing in the ventrolateral medulla, a column of $\mathrm{TH}+$ neurons located laterally to the posterior most part of the $\mathrm{C} 1$ nucleus and extending to the spinomedullary junction was designated as the A1 nucleus. The A1 column was distinguished from the ventrolateralC1 column by occupying a position lateral to the nucleus ambiguus, whereas the $\mathrm{C} 1$ nucleus was located medial to nucleus ambiguus. In the dorsal part of the medulla, in the region of the anterior part of the dorsal and medial border of the nucleus tractus solitarius, a distinct 
moderately dense cluster of $\mathrm{TH}+$ neurons was designated as the $\mathrm{C} 2$ nucleus. Within this nucleus there was a clear region close to the floor of the fourth ventricle termed the dorsal strip and a continuation of this cluster into the region of the tractus solitarius termed the rostral subdivision of the $\mathrm{C} 2$ nucleus. Between the caudal portions of the dorsal motor vagus and hypoglossal cranial nerve nuclei, a small number of $\mathrm{TH}+$ neurons represented the A2 nucleus. Some of these A2 neurons were located a small distance into the dorsal caudal medullary tegmentum. Straddling the midline, dorsal to the central canal and the dorsal motor vagus nucleus, and between the most caudal aspect of the bilateral C2 nucleus, was a single large, densely packed, cluster of intensely stained TH+neurons, the area postrema.

\subsection{Serotonergic nuclei}

The serotonergic nuclei $(5 \mathrm{HT}+)$ identified in the brains of all three species of this study were found to be the same as other eutherian mammals studied to date (Steinbusch, 1981; Maseko et al., 2007; Dell et al., 2010; Kruger et al., 2012). These nuclei were all located within the brainstem and can be divided into a rostral and caudal cluster. Both of these clusters contained distinct nuclei found throughout the brainstem from the level of the decussation of the superior cerebellar peduncle through to the spinomedullary junction. All three species examined exhibited the same complement of serotonergic nuclei in both the rostral and caudal clusters.

\subsubsection{Rostral cluster}

Within the rostral cluster we found evidence for the caudal linear nucleus (CLi), the supralemniscal nucleus (B9), the median raphe nucleus (MnR) and the dorsal raphe complex formed of six distinct nuclei (Figs. 2K-L, 3K-M, 4M-O, 8). The CLi nucleus was the most rostral of the serotonergic nuclei found and the 5HT+ neurons formed a moderate density cluster around the midline immediately dorsal to the interpeduncular nucleus in a location just anterior to the decussation of the superior cerebellar peduncle in all three species. The serotonergic neurons forming the B9 nucleus appeared to be a lateral extension of the most ventral portion of CLi. The $5 \mathrm{HT}+\mathrm{B} 9$ neurons were found in a low density caudal to the A9pc (see above) and extended as an arc of neurons into the lateral and ventrolateral portion of the midbrain tegmentum. The 
median raphe nucleus $(\mathrm{MnR})$ was characterised by two distinct, densely packed 5HT+ neuronal columns on either side of the midline in a para-raphe position. The rostral border of this nucleus was coincident with the level of the decussation of the superior cerebellar peduncle and the caudal border of this nucleus was found at the level of the trigeminal motor nucleus.
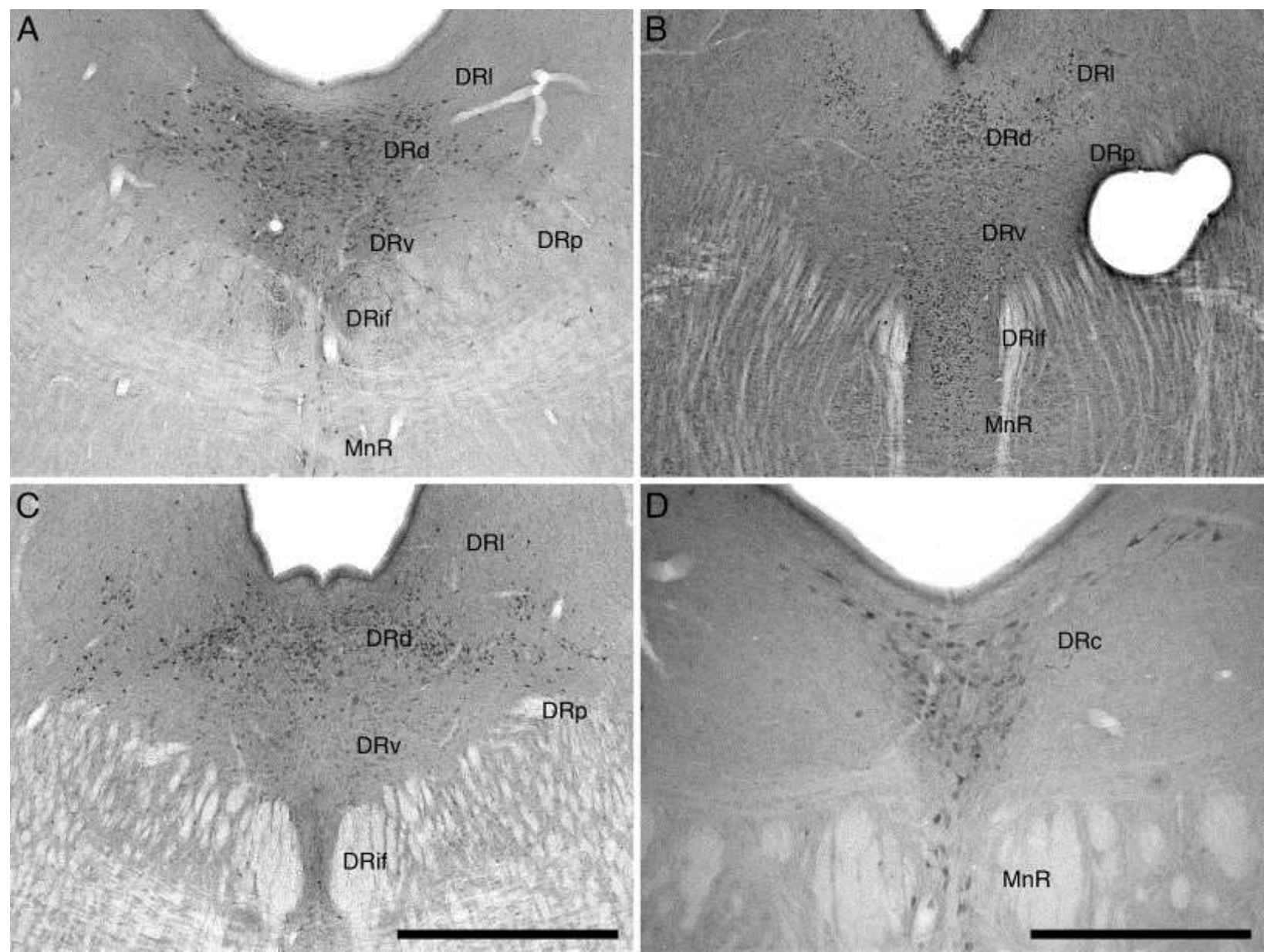

Figure 8: Photomicrographs showing neuronal groups that were immunopositive for serotonin in the brains of the Afrotherians studied. The dorsal raphe complex is shown for the giant otter shrew (A), the Hottentot golden mole (B) and the four-toed sengi (C). Scale bar in $\mathbf{C}=1000 \mu \mathrm{m}$ and applies to $\mathbf{A}, \mathbf{B}$ and $\mathbf{C}$. D. The caudal nucleus of the dorsal raphe (DRc) and the median raphe nucleus $(\mathbf{M n R})$ of the giant otter shrew. Scale bar in $\mathbf{D}=500 \mu \mathrm{m}$. See list for abbreviations.

Within the dorsal raphe nuclear complex we identified six distinct nuclei in all three species: the dorsal raphe interfascicular nucleus (DRif), the dorsal raphe ventral nucleus (DRv), the dorsal raphe dorsal nucleus (DRd), the dorsal raphe lateral nucleus (DRl), the dorsal raphe peripheral nucleus (DRp) and the dorsal raphe caudal nucleus (DRc). These six nuclei were 
found, for the most part, within the periaqueductal and periventricular grey matter from the level of the oculomotor nucleus to the trigeminal motor nucleus. Two pararaphe columns of 5HT+ cells located between the bilaterally paired medial longitudinal fasciculi represent the DRif nucleus in all three species. The DRv was found immediately dorsal to the DRif and just caudal to the oculomotor nuclei. The DRv exhibited a high density of 5HT+neurons. Immediately dorsal toDRv and ventral to the inferior border of the cerebral aqueduct a high-density cluster of 5HT+ neurons was designated as the DRd nucleus. A moderate density of 5HT+ neurons representing the DRp, were located in the ventrolateral portion of the periaqueductal grey matter lateral to the DRd and DRv. Some neurons of the DRp were found in the adjacent midbrain tegmentum and were the only ones found outside the periaqueductal grey matter. The5HT+ neurons of the DRl were located dorsolateral to the DRd and adjacent to the ventrolateral edges of the cerebral aqueduct in a low to moderate density. The neurons of this nucleus were readily distinguishable from the remainder of the dorsal raphe nuclei since they were substantially larger. As we followed the DRl caudally, where the cerebral aqueduct opened into the fourth ventricle and the DRd, DRv and DRif dissappeared, the neurons of the DRl formed an arc across the midline of the dorsal portion of the periventricular grey matter. This caudal arc of the DRl was classified as the DRc nucleus. We classified this as anindependent nucleus due to the lack of 5HT+ neurons in this region in the brain of monotremes (Manger et al., 2002b; Maseko et al., 2007; Dell et al., 2010; Kruger et al., 2012).

\subsubsection{Caudal cluster}

Within the caudal cluster we found evidence for the raphe magnus (RMg), rostral and caudal ventrolateral (RVL and CVL), raphe pallidus ( $R P a)$ and raphe obscurus (ROb) nuclei (Figs. 2M-P, 3M-P, 4O-S). The RMg was observed to be two columns of loosely aggregated moderate to large 5HT+ neurons located on either side of the midline from the level of the caudal pole of the trigeminal motor nucleus to the caudal pole of the facial nucleus. Within the left and right ventrolateral medullary tegmentum a distinct anteroposterior column of 5HT+ neurons extending from the level of the facial nucleus to the spinomedullary junction were observed. These have previously been termed the rostral and caudal ventrolateral serotonergic columns (e.g. Maseko et al., 2007; Moon et al., 2007; Dwarika et al., 2008). The RVL began as a lateroventral continuation of 5HT+ neurons from the lower portion of the RMg extending over 
the pyramidal tracts and consolidating as a distinct column lateral to the inferior olives. The inferior olive topologically distinguishes left and right RVL, and at the approximate level of nucleus ambiguus the RVL becomes the CVL. The CVL continues in the caudal ventrolateral medullary tegmentum until the spinomedullary junction is reached. Although the RVL and CVL were continuous in the species studied, and indeed several other eutherian mammals previously studied (e.g. Maseko et al., 2007; Moon et al., 2007; Dwarika et al., 2008; Dell et al., 2010; Kruger et al., 2012), we distinguish two components of these ventrolateral columns, as the caudal portions have not been reported in the opossum or the monotremes (Crutcher and Humbertson, 1978; Manger et al., 2002b). The 5HT+ neurons forming the RPa nucleus were found in the ventral midline of the medulla associated with the pyramidal tracts. These neurons were for the most part located between the two pyramidal tracts. Two loosely arranged bilateral columns of $5 \mathrm{HT}+$ neurons located either side of the midline from the level of nucleus ambiguus to the spinomedullary junction were classified as the ROb.

\subsection{Orexinergic (hypocretinergic) nuclei}

Orexin-A immunohistochemistry was used to identify orexin-A immunopositive neurons $(\mathrm{OrX}+)$ cells in the giant otter shrew and the four-toed sengi. The vast majority of orexin-A immunopositive neurons (OrX+) identified in the brains of these species were localised to the hypothalamus. Within the area where orexinergic neurons were located we could readily divide them into three distinct clusters: a main cluster (Mc), a zona incerta cluster (Zic) and an optic tract cluster (Otc) (Figs. 2G-H, 4I-K). In both species the main cluster (Mc) was identified as a large group of densely packed OrX+ neuronal cell bodies located lateral to the third ventricle in the perifornical region, with a moderate number of neuronal cell bodies extending medially from this area into the dorsomedial hypothalamus and a larger number extending into the lateral hypothalamic areas (Fig. 9). From the main cluster a group of OrX+ neuronal cell bodies extended laterally into the region of the zona incerta (Zic). This cluster had a very low density of OrX+ neurons that were mixed with neurons of the lateral hypothalamic cholinergic nucleus and the A13 nucleus of the catecholaminergic system (see above). The third cluster, the optic tract cluster (Otc) extended ventrolaterally from the main cluster to the ventrolateral region of the hypothalamus adjacent to the optic tract. This cluster exhibited a moderate density of OrX+ 
neuronal cell bodies. In both species the orexinergic neurons were typically bipolar in nature and exhibited no clear dendritic orientation, except for those in the Zic where the dendrites were observed to run parallel to the inferior border of the zona incerta.

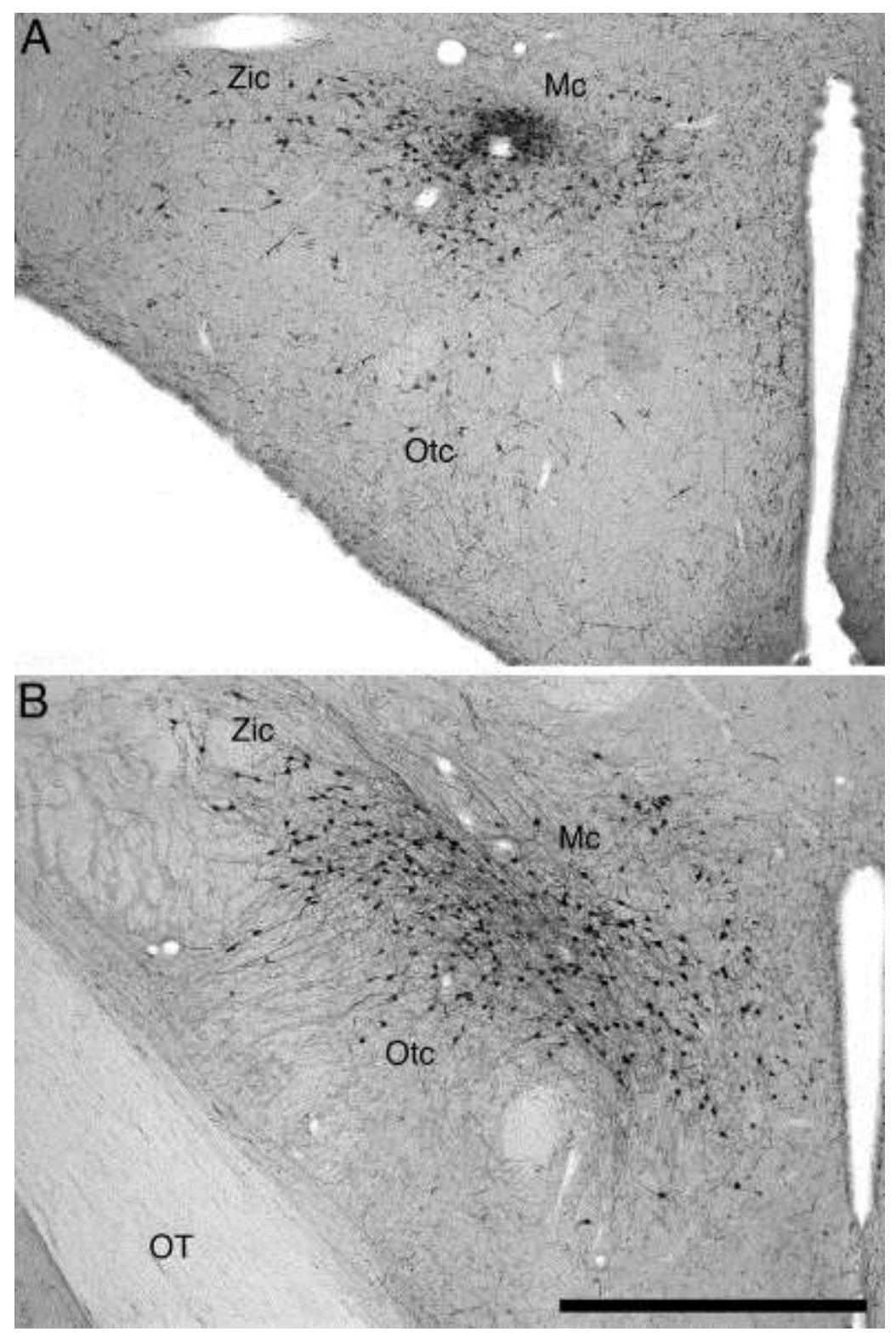

Figure 9: Photomicrographs showing hypothalamic neuronal groups that were immunoreactive for orexin-A in the brain of the giant otter shrew (A) and four-toed sengi (B). Mc - main orexinergic cluster; OT - optic tract; Otcoptic tract orexinergic cluster; Zic - zona incerta orexinergic cluster. Scale bar in $\mathbf{B}=1000 \mu \mathrm{m}$ and applies to both. 


\section{Discussion}

The nuclear organization and complement of the neural systems investigated in the current study within the brains of three previously unstudied species of Afrotheria, the giant otter shrew (Potomogale velox), the hottentot golden mole (Amblysomus hottentotus), and the fourtoed sengi (Petrodromus tetradactylus), were, for the most part, similar to that observed in many eutherian mammals previously studied (e.g. Dell et al., 2010). Despite this there were differences of note that may be related either to the phylogenetic history or current life histories of these species. The golden mole exhibited an extensive and divergent distribution of cholinergic interneurons, far beyond that seen in other mammals. The four-toed sengi also exhibited cholinergic neurons in regions of the brain not normally associated with the cholinergic system, but cholinergic neurons were previously observed in these regions in the closely related eastern rock elephant shrew (Pieters et al., 2010). In agreement with previous observations in the eastern rock elephant shrew (Pieters et al., 2010), we could not find evidence for the catecholaminergic dorsal division of the anterior hypothalamic group (A15d) in the four-toed sengi. Lastly, in both the golden mole and the four-toed sengi, the catecholaminergic diffuse division of the locus coeruleus (A6d) was made up of a small number of neurons, similar to that observed in the rock hyrax (Gravett et al., 2009). In contrast to these observations, the giant otter shrew appears to have a nuclear organization of these systems that can be described as very typical for a eutherian mammal.

\subsection{The "hypercholinergic" golden mole}

While the cholinergic system in the three species studied was mostly similar to that observed in other eutherian mammals, the golden mole was observed to have cholinergic interneurons throughout the cerebral neocortex and piriform cortex, the olfactory bulb, amygdala and hippocampus. The existence of cholinergic interneurons in this wide variety of locations has not been previously described. Cholinergic interneurons in the cerebral neocortex have only been observed previously in Murid rodents, with non-Murid rodents lacking these neurons (Bhagwandin et al., 2006; Kruger et al., 2012). Studies of the chemical nature of the cholinergic neocortical neurons in Rattus norvegicus, a Murid rodent, have indicated that these neurons likely co-contain vasoactive intestinal polypeptide (VIP) and gamma-aminobutyric acid (GABA) 
(Eckenstein and Baughman, 1984; Bayraktar et al., 1997). Thus, these neocortical neurons have multiple transmission lines that may allow them to modulate the cortical microcirculation in relation to these multiple transmission lines (Chedotal et al., 1994). In the case of the golden mole, this may indicate that the microcirculation undergoes a great deal of local control in many brain regions. The question remains as to why this may occur in the golden moles. Our observations show that the olfactory system of the golden mole is likely to be the dominant sense, and this is reflected in the large size of the olfactory bulb and the impressive size and modularization of the olfactory tubercle in comparison to the other species studied herein and previously. The cholinergic neurons found in the olfactory bulb, piriform cortex, and amygdala, all indicate the importance of the modulation of neuronal function and microcirculation in regions of the brain associated with olfaction. While the hippocampus and neocortex are not parts of the brain normally considered to be involved in processing olfactory information, it is likely, given the dominance of the olfactory system in the golden mole, that they are recruited for this purpose, perhaps in the recognition of location in relation to olfactory stimuli for the hippocampus, and in the process of decision making in relation to olfactory stimuli for the cerebral neocortex. While these ideas are obviously speculative, it would be of interest to examine these functional possibilities further in the hottentot golden mole, and to examine more species of golden moles to determine whether these supernumerary cholinergic neurons are a phylogenetically distinct characteristic of golden moles (the order Chrysochloridae), or whether they are restricted to the single species studied herein.

\subsection{Additional cholinergic neurons in the four-toed sengi}

In addition to the supernumerary cholinergic neurons observed in the golden mole, we observed cholinergic neurons in the superior and inferior colliculi and the cochlear nucleus in the four-toed sengi. Similar cholinergic neurons, in the same locations, were previously observed in the eastern rock elephant shrew (Pieters et al., 2010), both species being members of the order Macroscelididae, the elephant shrews or sengis. This similarity in the distribution of cholinergic neurons outside of the classically defined cholinergic system (Woolf, 1991) indicates a common ancestry for these species, and the evolution of these neural traits during the establishment of the Macroscelididae order (Manger, 2005). Thus, while we can conclude that the cholinergic neurons in these regions of the elephant shrew brain are likely to be a feature common to all 
elephant shrews, it is still possible that they play an important functional role related to aspects of the life-history of elephant shrews. As mentioned above, these cholinergic interneurons are likely to be involved in the modulation of the microcirculation in the brain regions in which they are found. In both species of elephant shrews studied, all three regions of the brain in which the cholinergic interneurons were found are related to audition. Both species of elephant shrews studied are known to produce species-specific patterns of foot-drumming behaviour in response to agonistic encounters (Faurie et al., 1996; Skliba et al., 2008), with most species of elephant shrews likely to show some form of foot drumming communication (Faurie et al., 1996). The drumming sounds created by the elephant shrews are detectable both via aerial and seismic transmission as a form of sound wave. The modulation of the frequency tuning circuits in the cochlear nuclei and spatial location circuits in the inferior colliculus may enhance the detection and location of the source of the drumming. The cholinergic neurons in the superior colliculus may enhance the visual targeting of the drumming source. Thus, it is possible that the common ancestor of elephant shrews used drumming for conspecific communication, augmented by cholinergic neurons in regions of the brain of importance for the detection and location of these auditory signals. These traits would then have been passed on to all descendents of the common ancestor and we see them in the brains of extant elephant shrews. Further exploration of the occurrence of these neurons across elephant shrew species, with specific reference to the speciesspecific pattern and behavioural use of foot drumming may provide data of interest to our understanding of the elephant shrews.

\subsection{Differences in the catecholaminergic systems and phylogenetic relationships}

We could not find evidence for the existence of the A15d nucleus in the four-toed sengi. In a previous study of the eastern rock elephant shrew, this nucleus was also absent (Pieters et al., 2010), indicating an order specific absence of this nucleus in the Macroscelididae (Manger, 2005). This particular catecholaminergic nucleus has been associated with seasonal control of reproduction (Thiery et al., 1989; Beccavin et al., 1998), and while the elephant shrews are seasonal breeders (Medger et al., 2012) there are indications that they are spontaneous ovulators that may breed opportunistically when conducive conditions arise (de Bruin and Bennett, pers. comm.). Thus, the release of inhibition of pulsatile luteinizing hormone, may allow opportunistic breeding opportunities for these mammals. In addition to the lack of the A15d nucleus, in both 
the foru-toed sengi and the golden mole, the number of neurons forming the diffuse portion of the locus coeruleus (A6d) was small in number. This characteristic is shared by the rock hyrax (Gravett et al., 2009), but not by the giant otter shrew. Thus, it appears that the common ancestor of the Macroscelididae, Chrysochloridae and Hyracoidea may have had this trait. While this concept is supported for the Macroscelididae and Chrysochloridae, to the exclusion of the Tenrecidea, due to the closer relationship of the Macroscelididae and Chrysochloridae, the occurrence of this trait in the Hyracoidea indicates, based on current understandings of the phylogenetic relationships of the Afrotheria (Arnason et al., 2008; Asher et al., 2009), that either this character evolved twice, or was lost in the Tenrecidea. Further comparative work on the anatomy of the locus coeruleus in other members of the Afrotheria will resolve this problem.

\subsection{The lack of changes in the serotonergic and orexinergic systems}

Previous studies of the nuclear organization of the serotonergic system across mammals have shown that the organization of this system is very conservative in terms of evolutionary changes, with all eutherian mammals studied to date showing a similar nuclear complement (Dell et al., 2010). The three Afrotherian species investigated in the current study do not negate these previous observations on the organization of the serotonergic system. The orexinergic system also appears to have a quite conservative organization across mammalian species studied to date. As with the two species of Afrotherian studied herein, the giant otter shrew and the four-toed sengi, most mammals have three specific clusters of orexinergic neurons, all located in the hypothalamus - a main cluster, an optic tract cluster and a zona incerta cluster. Three exceptions to this organization have been reported in the literature. Both hamsters and microchiropterans appear to lack the optic tract cluster of orexinergic neurons (Mintz et al., 2001; McGranaghan and Piggins, 2001; Korooshi and Klingenspor, 2005; Vidal et al., 2005; Kruger et al., 2010), while the cetartiodactyla, represented by the giraffe and harbour porpoise, are reported to have an additional cluster of parvocellular orexinergic neurons in the medial hypothalamus (Dell et al., 2012). Thus, for the most part, the organization of the orexinergic system is also quite conservative across mammals. 


\section{Acknowledgments}

This work was supported by funding from the South African National Research Foundation (PRM and NCB), the Belgian co-operation service at the Royal Museum for Central Africa (EG), and by a fellowship within the Postdoctoral-Program of the German Academic Exchange Service, DAAD (NP).

\section{References}

Arnason, U., Adegoke, J.A., Gullberg, A., Harley, E.H., Janke, A., Kullberg, M., 2008. Mitogenomic relationships of placental mammals and molecular estimates of their divergences. Gene 421, 37-51.

Asher, R.J., Maree, S., Bronner, G., Bennett, N.C., Bloomer, P., Czechowski, P., Meyer, M., Hofreiter, M., 2010. A phylogenetic estimate for golden moles (Mammalia, Afrotheria, Chrysochloridae). BMC Evol. Biol. 10, 69.

Bayraktar, T., Staiger, J.F., Acsady, L., Cozzari, C., Freund, T.F., Zilles, K., 1997. Colocalization of vasoactive intestinal polypeptide, gamma-aminobutyric acid and choline acetyltransferase in the neocortical interneurons of the adult rat. Brain Res. 757, 209-217.

Beccavin, C., Malpaux, B., Tillet, Y., 1998. Effect of oestradiol and photoperiod on TH mRNA concentrations in A15 and A12 dopamine cell groups in the ewe. J. Neuroendocrinol. 10, 59-66.

Bhagwandin, A., Fuxe, K., Manger, P.R., 2006. Choline acetyltransferase immunoreactive cortical interneurons do not occur in all rodents: a study of the phylogenetic occurrence of this neural characteristic. J. Chem. Neuroanat. 32, 208-216.

Bhagwandin, A., Fuxe, K., Bennett, N.C., Manger, P.R., 2008. Nuclear organization and morphology of cholinergic, putative catecholaminergic and serotonergic neurons in the brains of two species of African mole-rat. J. Chem. Neuroanat. 35, 371-387.

Bhagwandin, A., Fuxe, K., Bennett,N.C., Manger, P.R., 2011.Distribution of orexinergic neurons and their terminal networks in the brains of two species of African mole rats. J.Chem. Neuroanat. $41,32-42$. 
Chedotal, A., Cozzari, C., Faure, M.P., Hartman, B.K., Hamel, E., 1994. Distinct choline acetyltransferase (ChAT) and vasoactive intestinal polypeptide (VIP) bipolar neurons project to local blood vessels in the rat cerebral cortex. Brain Res. 646, 181-193.

Crutcher, K.A., Humbertson, A.O., 1978. The organization of monoamine neurons within the brainstem of the North American opossum (Didelphis virginiana). J. Comp. Neurol. 179, 195222.

Dahlström, A., Fuxe, K., 1964. Evidence for the existence of monoamine-containing neurons in the central nervous system. I. Demonstration of monoamine in the cell bodies of brainstem neurons. Acta Physiol. Scand. 62, 1-52.

Dell, L-A., Kruger, J-L., Bhagwandin, A., Jillani, N.E., Pettigrew, J.D., Manger, P.R., 2010. Nuclear organization of cholinergic, putative catecholaminergic and serotonergic systems in the brains of two megachiropteran species. J. Chem. Neuroanat. 40, 177-195.

Dell, L.A., Patzke, N., Bhagwandin, A., Bux, F., Fuxe, K., Barber, G., Siegel, J.M., Manger, P.R., 2012. Organization and number of orexinergic neurons in the hypothalamus of two species of Cetartiodactyla: a comparison of giraffe (Giraffa camelopardalis) and harbour porpoise (Phocoena phocoena). J. Chem. Neuroanat. 44, 98-109.

Dumbacher, J.P., Rathbun, G.B., Smit, H.A., Eiseb, S.J., 2012. Phylogeny and taxonomy of the round-eared sengis or elephant-shrews, genus Macroscelides (Mammalia, Afrotheria, Macroscelidea). PLoS One 7, e32410.

Dwarika, S., Maseko, B.C., Ihunwo, A.O., Fuxe, K., Manger, P.R., 2008. Distribution and morphology of putative catecholaminergic and serotonergic neurons in the brain of the greater canerat, Thryonomys swinderianus. J. Chem. Neuroanat. 35, 108-122.

Eckenstein, F., Baughman, R.W., 1984. Two types of cholinergic innervation in the cortex, one co-localised with vasoactive intestinal polypeptide. Nature 309, 153-155.

Faurie, A.S., Dempster, E.R., Perrin, M.R., 1996. Foot drumming patterns of southern African elephant-shrews. Mammalia 60, 567-576.

Gallyas, F., 1979.Silver staining of myelin by means of physical development.Neurolog. Res. 1, 
Gravett, N., Bhagwandin, A., Fuxe, K., Manger, P.R., 2009. Nuclear organization and morphology of cholinergic, putative catecholaminergic and serotonergic neurons in the brain of the rock hyrax, Procavia capensis. J. Chem. Neuroanat. 38, 57-74.

Gravett, N., Bhagwandin, A., Fuxe, K., Manger, P.R., 2011. Distribution of orexin-A immunoreactive neurons and their terminal networks in the brain of the rock hyrax, Procavia capensis. J. Chem. Neuroanat. 41, 86-96.

Hallström, B.M., Janke, A., 2008. Resolution among major placental mammal interordinal relationships with genome data imply that speciation influenced their earliest radiations. BMC Evol. Biol. 8, 162.

Hökfelt, T., Johansson, O., Fuxe, K., Goldstein, M., Park, D., 1976. Immunohistochemical studies on the localization and distribution of monoamine neuron systems in the rat brain. I. Tyrosine hydroxylase in the mes- and diencephalon. Med. Biol. 54, 427-53.

Hökfelt, T., Martenson, R., Björklund, A., Kleinau, S., Goldstein, M., 1984. Distributional maps of tyrosine-hydroxylase-immunoreactive neurons in the rat brain, in: Björklund, A., Hökfelt, T. (Eds.), Handbook of Chemical Neuroanatomy, vol. 2. Classical Neurotransmitters in the CNS, part 1. Elsevier, Amsterdam, pp. 277-379.

Kitahama, K., Geffard, M., Okamura, H., Nagatsu, I., Mons, N., Jouvet, M., 1990.Dopamineand dopa-immunoreactive neurons in the cat forebrain with reference to tyrosine hydroxylaseimmunohistochemistry.Brain Res. 518, 83-94.

Kitahama, K., Sakamoto, N., Jouvet, A., Nagatsu, I., Pearson, J., 1996. Dopamine-betahydroxylase and tyrosine hydroxylase immunoreactive neurons in the human brainstem. J. Chem. Neuroanat. 10, 137-146.

Khorooshi, R.M., Klingenspor, M., 2005. Neuronal distribution of melanin-concentrating hormone, cocaine- and amphetamine regulated transcript and orexin B in the brain of the Djungarian hamster (Phodopus sungorus). J Chem Neuroanat. 29, 137-148. 
Kruger, J-L., Dell, L-A., Pettigrew, J.D., Manger, P.R., 2010. Cellular location and major terminal networks of the orexinergic system in the brains of five microchiropteran species. J. Chem. Neuroanat. 40, 256-262.

Kruger, J-L., Patzke, N., Fuxe, K., Bennett, N.C., Manger, P.R., 2012. Nuclear organization of cholinergic, putative catecholaminergic, serotonergic and orexinergic systems in the brain of the African pygmy mouse (Mus minutoides): organizational complexity is preserved in small brains. J. Chem. Neuroanat. 44, 45-56.

Limacher, A.M., Bhagwandin, A., Fuxe, K., Manger, P.R., 2008. Nuclear organization and morphology of cholinergic, putative catecholaminergic and serotonergic neurons in the brain of the Cape porcupine (Hystrix africaeaustralis): Increased brain size does not lead to increased organizational complexity. J. Chem. Neuroanat. 36, 33-52.

Manger, P.R., 2005. Establishing order at the systems level in mammalian brain evolution. Brain Res. Bull. 66, 282-289.

Manger, P.R., Fahringer, H.M., Pettigrew, J.D., Siegel, J.M., 2002a. Distribution and morphology of cholinergic neurons in the brain of the monotremes as revealed by ChAT immunohistochemistry. Brain Behav. Evol. 60, 275-297.

Manger, P.R., Fahringer, H.M., Pettigrew, J.D., Siegel, J.M., 2002b. Distribution and morphology of serotonergic neurons in the brain of the monotremes. Brain Behav. Evol. 60, 315332.

Maseko, B.C., Bourne, J.A., Manger, P.R., 2007. Distribution and morphology of cholinergic, putative catecholaminergic and serotonergic neurons in the brain of the Egyptian Rousette flying fox, Rousettus aegyptiacus. J. Chem. Neuroanat. 34, 108-127.

McCormack, J.E., Faircloth, B.C., Crawford, N.G., Gowaty, P.A., Brumfield, R.T., Glenn, T.C., 2012. Ultraconserved elements are novel phylogenetic markers that resolve placental mammal phylogeny when combined with species-tree analysis. Genome Res. 22, 746-754.

McGranaghan, P.A., Piggins, H.D., 2001. Orexin A-like immunoreactivity in the hypothalamus and thalamus of the Syrian hamster (Mesocricetus auratus) and Siberain hamster (Phodopus sungorus), with special reference to circadian structures. Brain Res. 904, 234-244. 
Medger, K., Chimimba, C.T., Bennett, N.C., 2012. Seasonal reproduction in the eastern rock elephant-shrew (Elephantulus myurus): to potential influence by rainfall or ambient temperature. J. Zool. In press.

Meister, B., Hökfelt, T., Steinbusch, H.W., Skagerberg, G., Lindvall, O., Geffard, M., Joh, T.H., Cuello, A.C., Goldstein, M., 1988. Do tyrosine hydroxylase-immunoreactive neurons in the ventrolateral arcuate nucleus produce dopamine or only L-dopa? J. Chem. Neuroanat. 1, 59-64.

Mintz, E.M., van Den Pol, A.N., Casano, A.A., Albers, H.E., 2001. Distribution of hypocretin (orexin) immunoreactivity in the central nervous system of Syrian hamsters (Mesocricetus auratus). J. Chem. Neuroanat. 21, 225-238.

Moon, D.J., Maseko, B.C., Ihunwo, A., Fuxe, K., Manger, P.R., 2007. Distribution and morphology of catecholaminergic and serotonergic neurons in the brain of the highveld gerbil, Tatera brantsii. J. Chem. Neuroanat. 34, 134-144.

Olson, L., Fuxe, K., 1972. Further mapping out of the central noradrenaline neurons systems: projections of the "subcoeruleus" area. Brain Res. 43, 289-295.

Pieters, R.P., Gravett, N., Fuxe, K., Manger, P.R., 2010. Nuclear organization of cholinergic, putative catecholaminergic and serotonergic nuclei in the brain of the eastern rock elephant shrew, Elephantulus myurus. J. Chem. Neuroanat. 39, 175-188.

Prasad, A.B., Allard, M.W., NISC Comparative Sequencing Program, Green, E.D., 2008. Confirming the phylogeny of mammals by use of large comparative sequence data sets. Mol. Biol. Evol. 25, 1795-1808.

Ruggiero, D.A., Anwar, M., Gootman, P.M., 1992. Presumptive adrenergic neurons containing phenylethanolamine $\mathrm{N}$-methyltransferase immunoreactivity in the medulla oblongata of neonatal swine.Brain Res. 583, 105-119.

Skliba, J., Sumbera, R., Chitaukali, W.N., 2008. Reactions to disturbances in the context of antipredatory behaviour in a solitary subterranean rodent. J. Ethol. 26, 249-254.

Smeets, W.J.A.J., González, A., 2000. Catecholamine systems in the brain of vertebrates: new perspectives through a comparative approach. Brain Res. Rev. 33, 308-379. 
Steinbusch, H.W.M., 1981. Distribution of serotonin-immunoreactivity in the central nervous system of the rat - cell bodies and terminals. Neuroscience. 6, 557-618.

Thiery, J.C., Martin, C.B., Tillet, Y., Caldani, M., Quentin, M., Jamain, C., Ravault, J.P., 1989. Role of hypothalamic catecholamines in the regulation of luteinizing hormone and prolcatin secretion in the ewe during seasonal anestrus. Neuroendocrinol. 49, 80-87.

Törk, I., 1990. Anatomy of the serotonergic system. Ann. N.Y. Acad. Sci. 600, 9-35.

van Dijk, M.A., Madsen, O., Catzeflis, F., Stanhope, M.J., de Jong, W.W., Pagel, M., 2001. Protein sequence signatures support the African clade of mammals. Proc. Natl. Acad. Sci. U.S.A. 98, 188-193.

Vidal, L., Blanchard, J., Morin, L.P., 2005. Hypothalamic and zona incerta neurons expressing hypocretin, but not melanin concentrating hormone, project to the hamster intergeniculate leaflet. Neuroscience 134, 1081-1090.

Weihe, E., Depboylu, C., Schultz, B., Schafer, M.K.H., Edien, L.E., 2006. Three types of tyrosine hydroxylase-positive CNS neurons distinguished by Dopa decarboxylase and VMAT2 co-expression. Cell. Mol. Neurobiol. 26, 659-678.

Woolf, N.J., 1991. Cholinergic systems in mammalian brain and spinal cord. Prog. Neurobiol. $37,475-524$. 